\title{
Inhaled corticosteroids and incident pneumonia in patients with asthma: Systematic review and meta-analysis
}

\author{
Vikas Bansal ${ }^{1}$, Muhammad A. Mangi ${ }^{1}$, Margaret M. Johnson ${ }^{2}$, Emir Festic ${ }^{2 *}$
}

${ }^{1}$ Research fellow, Pulmonary and Critical Care Medicine, Mayo Clinic, Jacksonville FL, ${ }^{2}$ Consultant, Pulmonary and Critical Care Medicine, Mayo Clinic, Jacksonville FL

\footnotetext{
${ }^{*}$ Corresponding author: festic.emir@mayo.edu

Tel.: + 19049563331

Fax.: + 19049532848
}

Received: 28 August 2015

Accepted: 4 November 2015

Key words: Asthma - Pneumonia Meta-analysis.
Objectives. To systematically review all available studies on inhaled corticosteroid use and incident pneumonia in asthma patients. Methods. We performed a literature search from January 1, 1993, through August 15, 2015, using PubMed, Medline, CENTRAL, EMBASE, Scopus, ISI, Regulatory Documents, Web of Science and manufacturers' web clinical trial registries with multiple search terms. We included studies that compared the risk of incident pneumonia among patients utilizing and not utilizing inhaled corticosteroids. We then summarized risk estimates into two random-effect meta-analyses; one including randomized controlled trials and another one including observational studies. Results. Fourteen studies were estimable; ten randomized controlled trials included 19,098 participants and four observational studies included 44,016 participants. There was no heterogeneity in randomized trials and summed risk ratio demonstrated the use of inhaled corticosteroids was protective of pneumonia; risk ratio $0.74,95 \% \mathrm{CI} 0.57$ to $0.95, \mathrm{p}=0.02$. On the contrary, observational studies showed summed odds ratio of 1.97 ; $95 \%$ CI 1.87to 2.07 , $\mathrm{p}<0.0001, \mathrm{I}^{2}=0 \%$, suggesting increased risk of pneumonia with use of inhaled corticosteroids in asthma patients. Conclusions. Inhaled corticosteroids are associated with decreased risk of incident pneumonia in patients with asthma based on meta-analysis of available randomized trials. Although observational studies in similar patients suggested higher risk of pneumonia, the inherent methodological limitations confer lower grade of confidence in these studies.

\section{Introduction}

Inhaled corticosteroids (ICS) are the most efficacious controller therapy for persistent asthma in all ages. ICS have been shown to modulate the airway inflammation underlying airway hypersensitivity to viral infections, allergens and irritants $(1,2)$, reduce asthma symptoms (3), and improve lung function and quality of life (3), by reducing the frequency and severity of exacerbations (4), and the risk of hospitalization (5). They may also decrease asthma mortality (6), and possibly attenuate loss of lung function in adults. The combination of an ICS and longacting beta agonists is commonly prescribed for patients with asthma and is the preferred treatment for patients whose asthma is not controlled by an ICS alone $(7,8)$.

Although ICS demonstrate a favorable risk profile with minimal serious adverse effects, cataracts $(9,10)$, and hyperglycemia (11-13), are identified consequences complicating their use. Since the Toward a 
Revolution in Chronic Obstructive Pulmonary Disease (COPD) Health (TORCH) trial (14), evidence has suggested that ICS use may be associated with an increased risk of pneumonia in patients with COPD (15-17). In contrast to COPD, several investigations failed to demonstrate an association between ICS use and the development of pneumonia in patients with asthma (18-22). Recently, McKeever et al. suggested an increased risk of pneumonia and lower respiratory tract infections (LRTI) in asthma patients utilizing ICS (23). However, this study lacked systematic and radiographic ascertainment of pneumonia, thus limiting the validity of the conclusions.

Although asthma is an independent risk factor for pneumonia (24-28), it is not clear whether ICS are further independently associated with an increased risk of pneumonia in people with asthma. Due to the conflicting results of prior investigations and their methodological limitations, we systematically reviewed the relevant medical literature and performed a meta-analysis to investigate the association of inhaled corticosteroids on the incidence of pneumonia in patients with asthma.

\section{Methods}

The review protocol was written by a senior investigator (E.F.) as a part of the Master's Program at Mayo Clinic Center for Clinical and Translational Science, CTSC 5740: Systematic Reviews and Meta-Analysis (http:// www.mayo.edu/ctsa/education/currentcourses-in-clinical-and-translational-science-at-mayo-graduate-school/mayo-graduate-school-course-descriptions) and was not publicly registered.

\section{Eligibility criteria}

The specific inclusion criteria for this systematic review were: (1) randomized con- trolled trials with minimum follow up of 4 weeks or an observational study with follow up for duration of hospitalization in participants with asthma, (2) use of any ICS medication alone or in combination with other medication as intervention versus a control group not using ICS, (3) diagnosis of incident pneumonia or lower respiratory tract infection (LRTI), or non-tuberculous mycobacterial pneumonia (NTM). Thus, reviewed studies included in our meta-analysis were RCTs and observational studies comparing the unadjusted risk of incident pneumonia (community acquired, LRTI, NTM) between patients on ICS and not on ICS. The minimal duration of exposure to ICS was not limited. Studies of patients with COPD were not eligible.

\section{Search strategy and study selection}

The search strategy was designed and conducted by a head reference librarian at Mayo Clinic, Rochester, MN. Two reviewers (V.B., M.A.M.) independently and in duplicate searched PubMed, Medline, CENTRAL, EMBASE, Scopus, Web of Science and manufacturers' web clinical trial registries (GlaxoSmithKline, AstraZeneca) using multiple search terms with no language restrictions, from January 1, 1993, through August 15, 2015. They screened all titles and abstracts identified by the preliminary library search to accrue potentially eligible studies. Then, the same reviewers independently assessed all selected full-text manuscripts for the eligibility. Disagreements regarding eligibility between 2 reviewers were resolved through consensus and after an input from a third reviewer (E.F.).

\section{Study characteristics and quality assessment}

In order to adhere to principles of sound methodological quality, we selected data col- 
lection forms for RCTs based on Cochrane Collaboration risk assessment tool. For each study, we ascertained the methods for randomization sequence, allocation concealment, and identified imbalances in baseline patient characteristics, which groups were blinded, study attrition rate, and if the analyses were conducted with intention to treat (ITT). We used terms "low risk" and "high risk" of bias at the study level instead of scoring. For observational studies we adopted Newcastle-Ottawa scales for cohort and case-control studies, as applicable. Quality assessments were done independently and discrepancies were achieved by consensus. At the outcome level, we assessed risk of bias by using GRADE profiler, version 3.6 (GRADE working group).

\section{Outcome measures}

Among all studies on ICS use in asthma, those which measured and reported pneumonia (including LRTI and NTM) were analyzed in detail. Pneumonia was reported as a safety or adverse effect in all RCTs; all except one of the observational studies (23) included a more systematic assessment for pneumonia, including radiographic confirmation.

\section{Data extraction}

Two reviewers (V.B. M.A.M.) independently reviewed and abstracted data on pneumonia incidence and ICS use for each eligible RCT and observational study of patients with asthma. If there were multiple reports stemming from a single specific study database, data from the study version that provided the most robust information on pneumonia were extracted with other contributing studies included in the bibliography. When specific data was missing, corresponding authors were contacted through email, maximum of two attempts for each author. Of four authors, two replied to the first email and one of these two was able to provide required information, while two others did not respond after two attempts. Reviewers sorted data separately in all stages of study selection, data extraction, and quality assessment. All discrepancies found between 2 reviewers were resolved with consensus and after inputs from other two authors.

\section{Quantitative data synthesis and sensitivity analysis}

We analyzed data in Review Manager Software, version 5.2 (Nordic Cochrane Center, Copenhagen, Denmark), to evaluate combined risk ratio (RR) for RCTs and odds ratio (OR) for observational studies (due to inclusion of three case-control studies) with respective $95 \%$ confidence intervals (CI) using a random-effects model. All reported $p$-values are 2-sided, with significance set at less than 0.05 . The statistical heterogeneity was assessed using the $\mathrm{I}^{2}$ statistic where values of $50 \%$ or more were considered as a substantial level of heterogeneity. Where substantial statistical heterogeneity was present, we explored additionally study characteristics and to determine a potential source of heterogeneity. The subgroup analysis was defined by RCTs versus observational studies. Sensitivity analyses were planned to explore the influences on effect size by: statistical models (fixed vs random effects), individual trials and cohort versus case-control studies.

\section{Results}

Initial library search identified 463 potentially relevant citations after removing duplicates in the EndNote (version X4). We excluded 430 articles after the title and abstract reviews. Eleven additional studies were identified through the reviews of web-based pharmaceutical clinical trial registries; of these, 4 were published and 7 
were unpublished. There were no disagreements between 2 reviewers at this stage. We then investigated why 7 latter studies were not included in our initial library search results and discovered that their published versions did not contain the specific term "pneumonia", which our search was based on. We subsequently performed full review of 44 studies; of those, 18 studies fulfilled the inclusion criteria for qualitative analysis and 14 of those were estimable and therefore included in 2 quantitative analyses. The flowchart is shown in Figure 1, study characteristics are shown in Tables 1 and 2 and reasons for excluded studies are shown in Table 3.

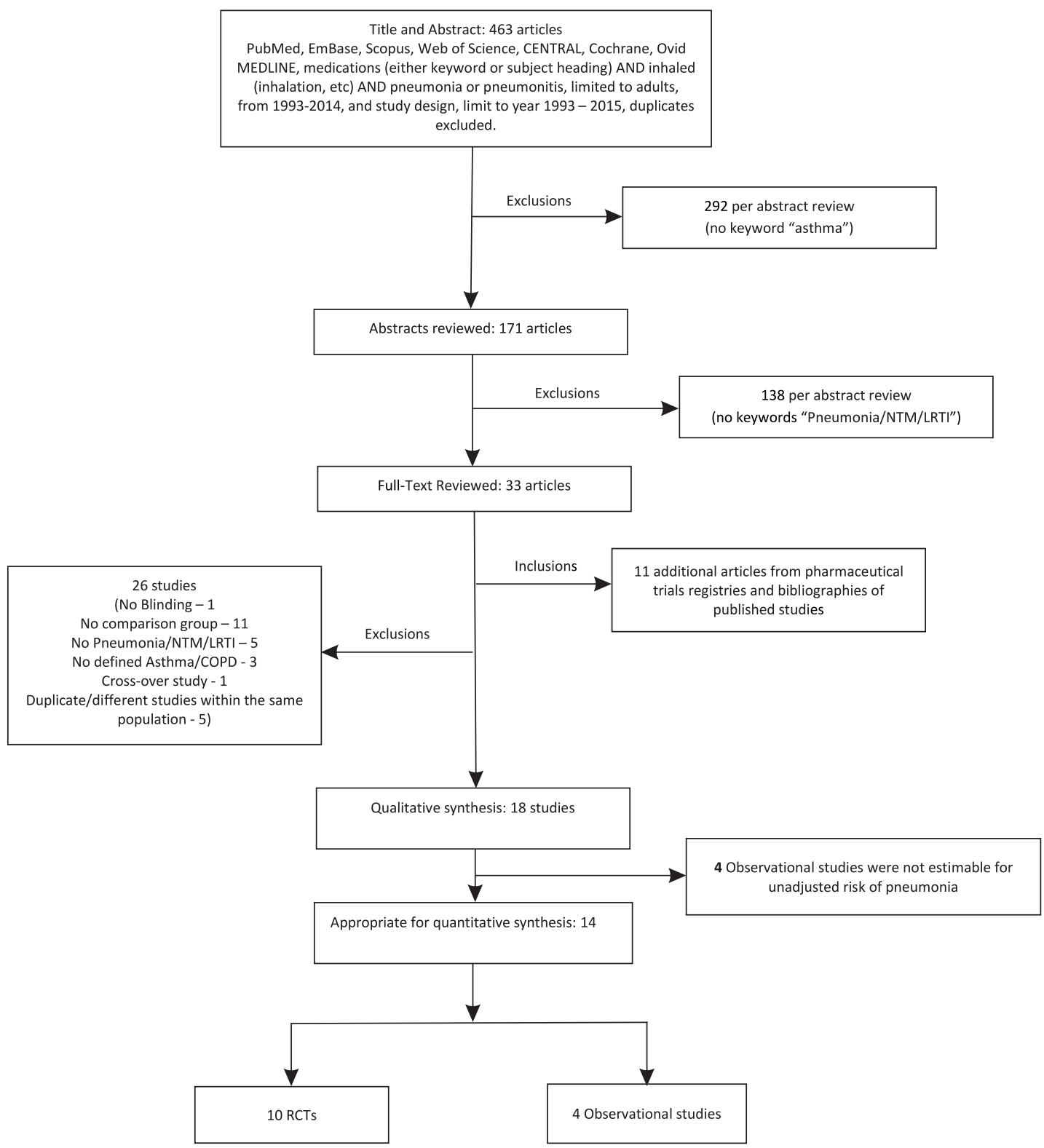

Figure 1 Study flow-chart. 
Table 1a Study characteristics for RCTs*

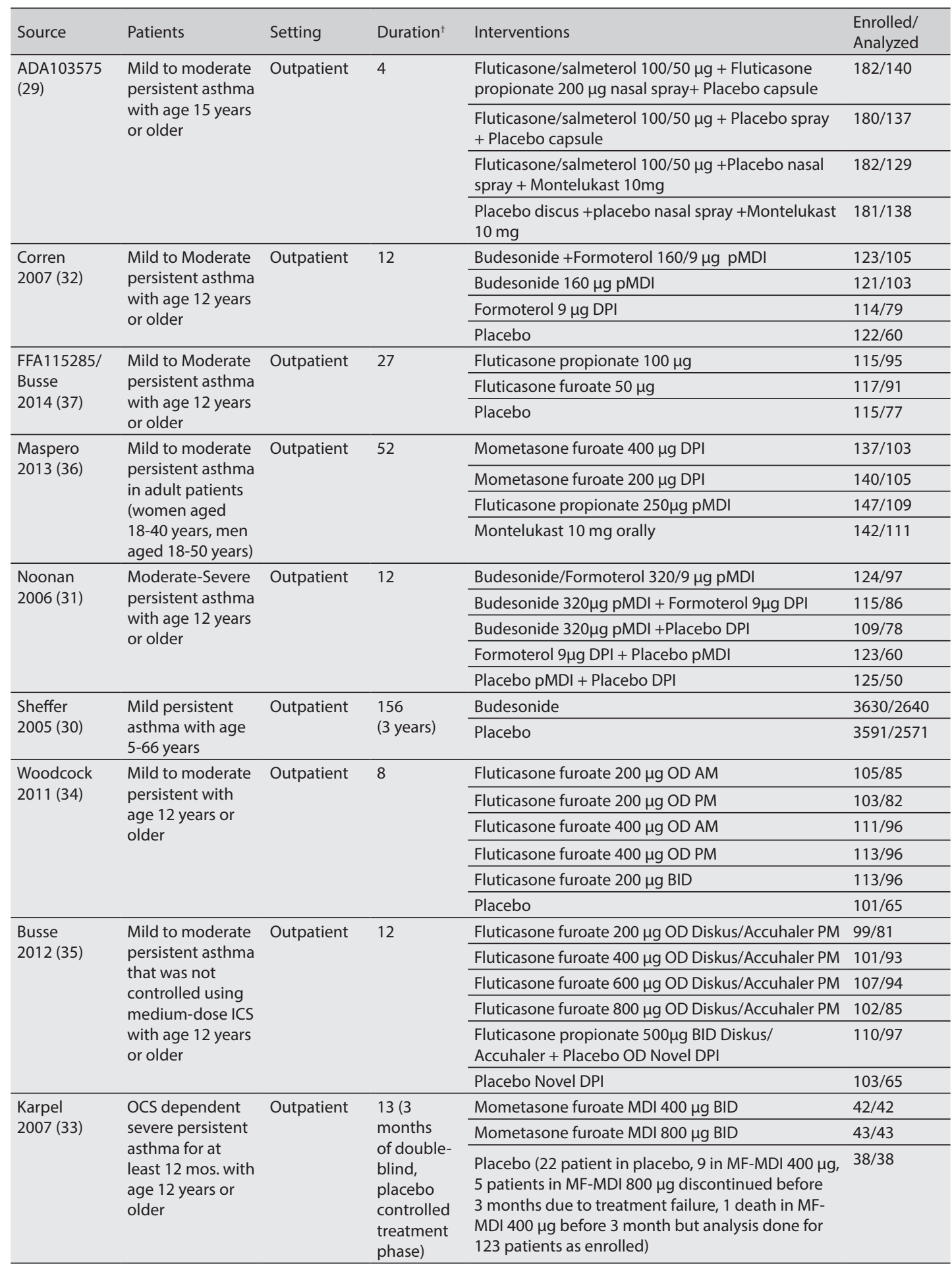

"Data on 26 unpublished RCTs from O'Byrne et al. (21) is not included in the table. ${ }^{\dagger}$ Duration in weeks; OCS=Ooral corticosteroids; pMDI=Delivered via metered-dose inhaler; $\mathrm{DPI}=$ Delivered via dry powder inhaler; $\mathrm{AM}=$ Morning dosing; $\mathrm{PM}=$ Evening dosing; $\mathrm{OD}=\mathrm{Once}$ daily; $\mathrm{BID}=\mathrm{Twice}$ daily. 
Table $1 \mathrm{~b}$ Quality assessment tables for RCTs*

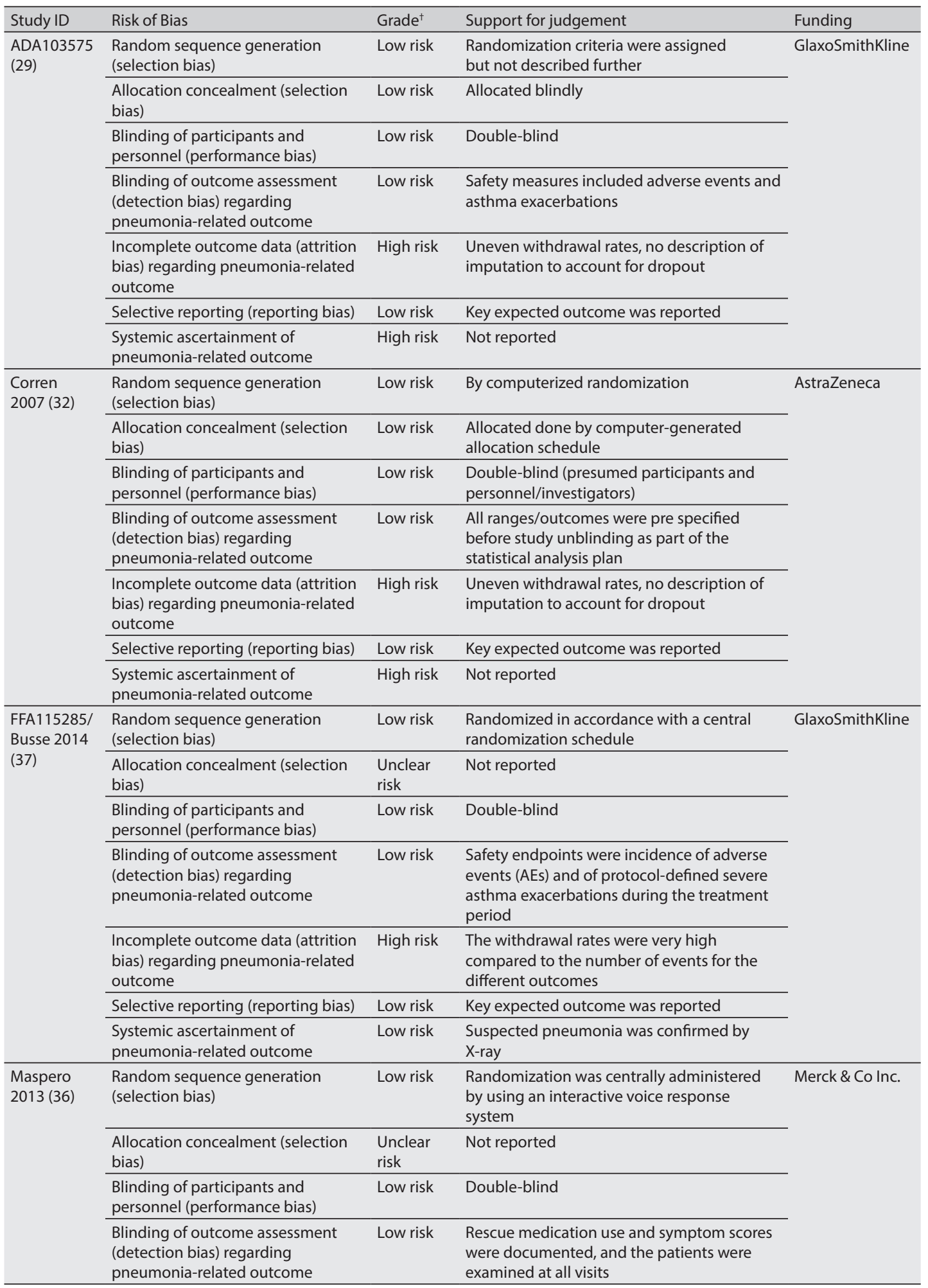


Continuation of Table $1 \mathrm{~b}$ Quality assessment tables for RCTs

\begin{tabular}{|c|c|c|c|c|}
\hline Study ID & Risk of Bias & Grade $^{\dagger}$ & Support for judgement & Funding \\
\hline \multirow[t]{3}{*}{$\begin{array}{l}\text { Maspero } \\
2013(36)\end{array}$} & $\begin{array}{l}\text { Incomplete outcome data (attrition } \\
\text { bias) regarding pneumonia-related } \\
\text { outcome }\end{array}$ & High risk & $\begin{array}{l}\text { The withdrawal rates were very high } \\
\text { compared to the number of events for the } \\
\text { different outcomes }\end{array}$ & Merck \& Co Inc. \\
\hline & Selective reporting (reporting bias) & Low risk & Key expected outcome was reported & \\
\hline & $\begin{array}{l}\text { Systemic ascertainment of } \\
\text { pneumonia-related outcome }\end{array}$ & High risk & Not reported & \\
\hline \multirow[t]{7}{*}{$\begin{array}{l}\text { Noonan } \\
2006(31)\end{array}$} & $\begin{array}{l}\text { Random sequence generation } \\
\text { (selection bias) }\end{array}$ & Low risk & $\begin{array}{l}\text { Randomization was performed using a } \\
\text { computer generated allocation schedule and } \\
\text { stratified by asthma severity, based on the } \\
\text { daily dose of ICS before entering the study }\end{array}$ & AstraZeneca \\
\hline & $\begin{array}{l}\text { Allocation concealment (selection } \\
\text { bias) }\end{array}$ & Low risk & Computer generated allocation & \\
\hline & $\begin{array}{l}\text { Blinding of participants and } \\
\text { personnel (performance bias) }\end{array}$ & Low risk & Double-blind & \\
\hline & $\begin{array}{l}\text { Blinding of outcome assessment } \\
\text { (detection bias) regarding } \\
\text { pneumonia-related outcome }\end{array}$ & Low risk & $\begin{array}{l}\text { Safety was evaluated based on adverse } \\
\text { events, laboratory evaluations, vital signs, } \\
\text { ECGs, 24-hour Holter monitoring and } \\
\text { physical examinations }\end{array}$ & \\
\hline & $\begin{array}{l}\text { Incomplete outcome data (attrition } \\
\text { bias) regarding pneumonia-related } \\
\text { outcome }\end{array}$ & High risk & $\begin{array}{l}\text { The withdrawal rates were very high } \\
\text { compared to the number of events for the } \\
\text { different outcomes }\end{array}$ & \\
\hline & Selective reporting (reporting bias) & Low risk & Key expected outcome was reported & \\
\hline & $\begin{array}{l}\text { Systemic ascertainment of } \\
\text { pneumonia-related outcome }\end{array}$ & High risk & Not reported & \\
\hline \multirow[t]{7}{*}{$\begin{array}{l}\text { Sheffer } \\
2005(30)\end{array}$} & $\begin{array}{l}\text { Random sequence generation } \\
\text { (selection bias) }\end{array}$ & Low risk & $\begin{array}{l}\text { Randomization was stratified into } \\
\text { two strata according to age; age less than } \\
11 \text { years or age at least } 11 \text { years } \\
\text { Within each stratum, patients were } \\
\text { randomized in blocks of ten, five in } \\
\text { each treatment group }\end{array}$ & AstraZeneca \\
\hline & $\begin{array}{l}\text { Allocation concealment (selection } \\
\text { bias) }\end{array}$ & Low risk & Randomly allocated & \\
\hline & $\begin{array}{l}\text { Blinding of participants and } \\
\text { personnel (performance bias) }\end{array}$ & Low risk & Double-blind & \\
\hline & $\begin{array}{l}\text { Blinding of outcome assessment } \\
\text { (detection bias) regarding } \\
\text { pneumonia-related outcome }\end{array}$ & $\begin{array}{l}\text { Unclear } \\
\text { risk }\end{array}$ & $\begin{array}{l}\text { Safety outcomes of the START } \\
\text { clinical study included all AEs and asthma- } \\
\text { related events from spontaneous reporting } \\
\text { and patient's responses to standard } \\
\text { questioning during the } 3 \text {-year study period } \\
\text { ( } 6 \text { and } 12 \text { weeks after randomization and } \\
\text { then every } 3 \text { months up to } 3 \text { years) }\end{array}$ & \\
\hline & $\begin{array}{l}\text { Incomplete outcome data (attrition } \\
\text { bias) regarding pneumonia-related } \\
\text { outcome }\end{array}$ & High risk & $\begin{array}{l}\text { The withdrawal rates were very high } \\
\text { compared to the number of events for the } \\
\text { different outcomes }\end{array}$ & \\
\hline & Selective reporting (reporting bias) & Low risk & Key expected outcome was reported & \\
\hline & $\begin{array}{l}\text { Systemic ascertainment of } \\
\text { pneumonia-related outcome }\end{array}$ & High risk & Not reported & \\
\hline \multirow[t]{3}{*}{$\begin{array}{l}\text { Woodcock } \\
2011 \text { (34) }\end{array}$} & $\begin{array}{l}\text { Random sequence generation } \\
\text { (selection bias) }\end{array}$ & Low risk & $\begin{array}{l}\text { The central randomization schedule } \\
\text { was generated by the sponsor using } \\
\text { a validated computerized system }\end{array}$ & GlaxoSmithKline \\
\hline & $\begin{array}{l}\text { Allocation concealment (selection } \\
\text { bias) }\end{array}$ & Low risk & $\begin{array}{l}\text { Allocated randomly by using Registration } \\
\text { and Medication Ordering System (RAMOS), } \\
\text { an automated, interactive telephone based } \\
\text { system }\end{array}$ & \\
\hline & $\begin{array}{l}\text { Blinding of participants and } \\
\text { personnel (performance bias) }\end{array}$ & Low risk & Double-blind & \\
\hline
\end{tabular}


Acta Medica Academica 2015;44:135-158

Continuation of Table $1 \mathrm{~b}$ Quality assessment tables for RCTs

\begin{tabular}{|c|c|c|c|c|}
\hline Study ID & Risk of Bias & Grade $^{\dagger}$ & Support for judgement & Funding \\
\hline \multirow[t]{3}{*}{$\begin{array}{l}\text { Woodcock } \\
2011(34)\end{array}$} & $\begin{array}{l}\text { Blinding of outcome assessment } \\
\text { (detection bias) regarding } \\
\text { pneumonia-related outcome }\end{array}$ & Low risk & $\begin{array}{l}\text { The following safety endpoints were } \\
\text { evaluated: incidence of adverse events } \\
\text { (AEs) and serious AEs (SAEs), vital signs, } \\
\text { hematology, clinical chemistry, and urinalysis } \\
\text { parameters, oropharyngeal examinations, } \\
\text { and withdrawals due } \\
\text { to worsening asthma. AEs/SAEs were coded } \\
\text { using the Medical Dictionary } \\
\text { for Regulatory Activities }\end{array}$ & \multirow[t]{3}{*}{ GlaxoSmithKline } \\
\hline & $\begin{array}{l}\text { Incomplete outcome data (attrition } \\
\text { bias) regarding pneumonia-related } \\
\text { outcome }\end{array}$ & High risk & $\begin{array}{l}\text { The withdrawal rates were very high } \\
\text { compared to the number of events } \\
\text { for the different outcomes }\end{array}$ & \\
\hline & Selective reporting (reporting bias) & $\begin{array}{l}\text { Unclear } \\
\text { risk }\end{array}$ & $\begin{array}{l}\text { Authors used upper respiratory tract } \\
\text { infection and respiratory tract infection } \\
\text { separately in AE. We presumed RTI was LRTI }\end{array}$ & \\
\hline \multirow[t]{5}{*}{$\begin{array}{l}\text { Busse } \\
2012(35)\end{array}$} & $\begin{array}{l}\text { Random sequence generation } \\
\text { (selection bias) }\end{array}$ & Low risk & $\begin{array}{l}\text { The central randomization schedule was } \\
\text { generated by the sponsor using } \\
\text { a validated computerized system }\end{array}$ & \multirow[t]{5}{*}{ GlaxoSmithKline } \\
\hline & $\begin{array}{l}\text { Allocation concealment (selection } \\
\text { bias) }\end{array}$ & Low risk & $\begin{array}{l}\text { Allocated randomly by using Registration } \\
\text { and Medication Ordering System (RAMOS), } \\
\text { an automated, interactive telephone based } \\
\text { system }\end{array}$ & \\
\hline & $\begin{array}{l}\text { Blinding of participants and } \\
\text { personnel (performance bias) }\end{array}$ & Low risk & Double-blind & \\
\hline & Selective reporting (reporting bias) & Low risk & Key expected outcome was reported & \\
\hline & $\begin{array}{l}\text { Systemic ascertainment of } \\
\text { pneumonia-related outcome }\end{array}$ & High risk & Not reported & \\
\hline \multirow[t]{7}{*}{$\begin{array}{l}\text { Karpel } \\
2007(33)\end{array}$} & $\begin{array}{l}\text { Random sequence generation } \\
\text { (selection bias) }\end{array}$ & Low risk & $\begin{array}{l}\text { Randomization criteria were } \\
\text { assigned but not described further }\end{array}$ & \multirow{7}{*}{$\begin{array}{l}\text { No source of } \\
\text { funding/support } \\
\text { mentioned in } \\
\text { article }\end{array}$} \\
\hline & $\begin{array}{l}\text { Allocation concealment (selection } \\
\text { bias) }\end{array}$ & $\begin{array}{l}\text { Unclear } \\
\text { risk }\end{array}$ & Not reported & \\
\hline & $\begin{array}{l}\text { Blinding of participants and } \\
\text { personnel (performance bias) }\end{array}$ & Low risk & Double-blind & \\
\hline & $\begin{array}{l}\text { Blinding of outcome assessment } \\
\text { (detection bias) regarding } \\
\text { pneumonia-related outcome }\end{array}$ & Low risk & $\begin{array}{l}\text { All patients were monitored for } \\
\text { adverse events and changes in physical } \\
\text { findings, vital signs, hematological } \\
\text { and blood chemistry profiles, and } \\
\text { electrocardiographic profiles }\end{array}$ & \\
\hline & $\begin{array}{l}\text { Incomplete outcome data (attrition } \\
\text { bias) regarding pneumonia-related } \\
\text { outcome }\end{array}$ & High risk & $\begin{array}{l}\text { The withdrawal rates were very high } \\
\text { compared to the number of events for the } \\
\text { different outcomes }\end{array}$ & \\
\hline & Selective reporting (reporting bias) & Low risk & Key expected outcome was reported & \\
\hline & $\begin{array}{l}\text { Systemic ascertainment of } \\
\text { pneumonia-related outcome }\end{array}$ & High risk & Not reported & \\
\hline
\end{tabular}

*Data on 26 unpublished RCTs from O'Byrne et al. (21) is not included in the table; ${ }^{\dagger}$ Graded by authors; LRTI=Lower respiratory tract infection; $\mathrm{NTM}=$ Nontuberculous pulmonary mycobacteriosis / non-tuberculous mycobacterial pneumonia. 
Table 2a Study characteristics for observational studies

\begin{tabular}{|c|c|c|c|c|c|c|c|c|}
\hline Source & Type of study & Patients & Setting & Duration & Interventions & Subjects (n) & Risk of bias & \\
\hline \multirow{2}{*}{$\begin{array}{l}\text { Almirall } \\
2010(20)\end{array}$} & \multirow{2}{*}{$\begin{array}{l}\text { Case control } \\
\text { study }\end{array}$} & \multirow[b]{2}{*}{$\begin{array}{l}\text { Diagnosis of } \\
\text { community- } \\
\text { acquired } \\
\text { pneumonia patient } \\
\text { with three chronic } \\
\text { respiratory diseases } \\
\text { that require inhaled } \\
\text { therapy were } \\
\text { included: chronic } \\
\text { bronchitis, COPD } \\
\text { and asthma with } \\
\text { age } 14 \text { years or } \\
\text { older }\end{array}$} & \multirow[t]{2}{*}{ Outpatient } & \multirow{2}{*}{$\begin{array}{l}1 \text { year } \\
(1999-2000)\end{array}$} & Asthma ICS & 30 & Selection & Low \\
\hline & & & & & $\begin{array}{l}\text { Asthma } \\
\text { Non-ICS }\end{array}$ & 344 & Indication & High \\
\hline \multirow{2}{*}{$\begin{array}{l}\text { Andrejak } \\
2013(41)\end{array}$} & \multirow{2}{*}{$\begin{array}{l}\text { Population } \\
\text { based case- } \\
\text { control study }\end{array}$} & \multirow[b]{2}{*}{$\begin{array}{l}\text { Adult patient } \\
\text { (age } 15 \text { years } \\
\text { or older) with } \\
\text { microbiologically } \\
\text { confirmed NTM } \\
\text { pulmonary disease } \\
\text { with any chronic } \\
\text { respiratory diseases }\end{array}$} & \multirow[t]{2}{*}{ Outpatient } & \multirow{2}{*}{$\begin{array}{l}12 \text { years } \\
(1997-2008)\end{array}$} & Asthma ICS & 30 & Selection & Low \\
\hline & & & & & $\begin{array}{l}\text { Asthma } \\
\text { Non-ICS }\end{array}$ & 3 & Indication & High \\
\hline \multirow{2}{*}{$\begin{array}{l}\text { Festic } \\
2014(22)\end{array}$} & \multirow[t]{2}{*}{ Cohort study } & \multirow[b]{2}{*}{$\begin{array}{l}\text { Adult patients } \\
\text { hospitalized with } \\
\text { at least } 1 \text { major } \\
\text { risk factor for acute } \\
\text { respiratory distress } \\
\text { syndrome }\end{array}$} & \multirow[t]{2}{*}{ Inpatient } & \multirow{2}{*}{$\begin{array}{l}\text { Hospitalization } \\
\text { Mar. 2009-Aug. } \\
2009\end{array}$} & Asthma ICS & 149 & Selection & Low \\
\hline & & & & & $\begin{array}{l}\text { Asthma } \\
\text { Non-ICS }\end{array}$ & 291 & Indication & High \\
\hline \multirow{2}{*}{$\begin{array}{l}\text { Mckeever } \\
2013(23)\end{array}$} & \multirow{2}{*}{$\begin{array}{l}\text { Nested Case } \\
\text { control study }\end{array}$} & \multirow[b]{2}{*}{$\begin{array}{l}\text { Adult asthma } \\
\text { patients (age } \\
18 \text { to } 80 \text { ) with } \\
\text { pneumonia or } \\
\text { lower respiratory } \\
\text { tract infection }\end{array}$} & \multirow[t]{2}{*}{ Outpatient } & \multirow{2}{*}{$\begin{array}{l}3 \text { years } \\
(2004-2007)\end{array}$} & Asthma ICS & 15594 & Selection & High \\
\hline & & & & & $\begin{array}{l}\text { Asthma } \\
\text { Non-ICS }\end{array}$ & 27575 & Indication & High \\
\hline \multirow{2}{*}{$\begin{array}{l}\text { To M } \\
2004 \text { (19) }\end{array}$} & \multirow{2}{*}{$\begin{array}{l}\text { Retrospective } \\
\text { cohort study }\end{array}$} & \multirow[b]{2}{*}{$\begin{array}{l}\text { Asthma patients } \\
\text { who required } \\
\text { hospitalization } \\
\text { for community- } \\
\text { acquired } \\
\text { pneumonia with } \\
\text { age } 16 \text { year or older }\end{array}$} & \multirow[t]{2}{*}{ Inpatient } & \multirow{2}{*}{$\begin{array}{l}\text { Hospitalization } \\
13 \text { years } \\
(1989-2001)\end{array}$} & Asthma ICS & 37 & Selection & Low \\
\hline & & & & & $\begin{array}{l}\text { Asthma } \\
\text { Non-ICS }\end{array}$ & 25 & Indication & High \\
\hline \multirow{2}{*}{$\begin{array}{l}\text { Ferrer } \\
2014(42)\end{array}$} & \multirow{2}{*}{$\begin{array}{l}\text { Prospective } \\
\text { observational } \\
\text { cohort study }\end{array}$} & \multirow{2}{*}{$\begin{array}{l}\text { Patients aged } \geq 16 \\
\text { years hospitalized } \\
\text { with a diagnosis } \\
\text { of CAP }\end{array}$} & \multirow[t]{2}{*}{ Inpatient } & \multirow{2}{*}{$\begin{array}{l}\text { Hospitalization } \\
\text { Jan. 2003-Oct. } \\
2005\end{array}$} & Asthma ICS & 12 & Selection & Low \\
\hline & & & & & $\begin{array}{l}\text { Asthma } \\
\text { Non-ICS }\end{array}$ & 28 & Indication & High \\
\hline \multirow{2}{*}{$\begin{array}{l}\text { Sellares } \\
2013(40)\end{array}$} & \multirow{2}{*}{$\begin{array}{l}\text { Prospective } \\
\text { observational } \\
\text { cohort study }\end{array}$} & \multirow[b]{2}{*}{$\begin{array}{l}\text { Patients admitted } \\
\text { to the emergency } \\
\text { room with a } \\
\text { diagnosis of CAP } \\
\text { with age } \\
16 \text { year or older }\end{array}$} & \multirow[t]{2}{*}{ Inpatient } & \multirow{2}{*}{$\begin{array}{l}\text { Hospitalization } \\
\text { Jan. 1997-Jul. } \\
2008\end{array}$} & Asthma ICS & 81 & Selection & Low \\
\hline & & & & & $\begin{array}{l}\text { Asthma } \\
\text { Non-ICS }\end{array}$ & 72 & Indication & High \\
\hline \multirow{2}{*}{$\begin{array}{l}\text { Terraneo } \\
2014(43)\end{array}$} & & Adult patients & Inpatient & Hospitalization & Asthma ICS & 72 & Selection & Low \\
\hline & $\begin{array}{l}\text { observational } \\
\text { cohort study }\end{array}$ & $\begin{array}{l}\text { hospitalized with } \\
\text { CAP }\end{array}$ & & $\begin{array}{l}\text { in Jan. 2000- } \\
\text { Dec. } 2011\end{array}$ & $\begin{array}{l}\text { Asthma } \\
\text { Non-ICS }\end{array}$ & 67 & Indication & High \\
\hline
\end{tabular}

$\mathrm{COPD}=$ Chronic obstructive pulmonary disease; $\mathrm{ICS}=$ Inhaled corticosteroids; $C A P=C$ mmunity-acquired pneumonia. 
Table 2b Quality assessment tables for observational studies

Case Control studies

\begin{tabular}{llll}
\hline Study & Selection & Comparability & Exposure \\
\hline Almirall 2010 & $3 / 5$ & $1 / 2$ & $4 / 4$ \\
Andrejak 2013 & $3 / 5$ & $1 / 2$ & $3 / 4$ \\
Mckeever 2013 & $3 / 5$ & $1 / 2$ & $3 / 4$ \\
\hline
\end{tabular}

Note: Points assessed in lieu of actual over possible stars per Quality Assessment Scale used (Supplementary material).
Cohort studies

\begin{tabular}{llll}
\hline Study & Selection & Comparability & Outcome \\
\hline Ferrer 2014 & $3 / 5$ & $2 / 2$ & $3 / 3$ \\
Festic 2014 & $5 / 5$ & $2 / 2$ & $3 / 3$ \\
\hline Sellares 2013 & $3 / 5$ & $2 / 2$ & $3 / 3$ \\
\hline Terraneo 2014 & $3 / 5$ & $2 / 2$ & $3 / 3$ \\
To m 2004 & $3 / 5$ & $2 / 2$ & $3 / 3$ \\
\hline
\end{tabular}

Note: Points assessed in lieu of actual over possible stars per Quality Assessment Scale used (Supplementary material).

Table 3 Excluded studies

\begin{tabular}{|c|c|}
\hline Study ID & Reason for exclusion \\
\hline D589IL00001/NCT01232348 & No blinding, no control group \\
\hline Beasley 2015 & No control group \\
\hline D5890L00008/NCT00242411 & No control group \\
\hline D5890L00009/NCT00290264 & No control group \\
\hline Hojo 2012 & No control group \\
\hline Lin 2015 & No control group \\
\hline Lukaszyk 2011 & No control group \\
\hline Lukaszyk 2011-2 & No control group \\
\hline SAM 106538/NCT00363480 & No control group \\
\hline Peters SP 2010 & No control group \\
\hline Teichert 2014 & No control group \\
\hline Woodcock 2014 & No control group \\
\hline Corren 2013 & No pneumonia reported \\
\hline $\begin{array}{l}\text { HZA106827/ NCT01165138/Bleecker } \\
2014\end{array}$ & No pneumonia reported \\
\hline Nathan 2012 & No pneumonia reported \\
\hline Pearlman 2013 & No pneumonia reported \\
\hline Price 2013 & No pneumonia reported \\
\hline Cheng 2013 & Cross-over design \\
\hline Almirall 2008 & Duplicate publication, same study population as in Almirall 2010 \\
\hline Almirall 2013 & Duplicate publication, same study population as in Almirall 2010 \\
\hline $\begin{array}{l}\text { D5254C00111/NCT00641914/O'Byrne } \\
2009\end{array}$ & Duplicate publication, same study population as in Sheffer 2005 \\
\hline NCT01232335 & Duplicate publication, same study population as in D589IL00001/NCT01232348 \\
\hline Pauwels 2003 & Duplicate publication, same study population as in Sheffer 2005 \\
\hline Almirall 1999 & No distinction between Asthma versus COPD cases \\
\hline Eurich 2013 & No distinction between Asthma versus COPD cases \\
\hline Farr 2000 & No distinction between Asthma versus COPD cases \\
\hline
\end{tabular}




\section{Randomized controlled trials}

There were 9 RCTs (29-37) and one additional study (21) that reported results of 26 unpublished pharmaceutical trials on different formulations of budesonide compared to placebo. Together, these studies included 19,098 patients, of whom 12,008 received ICS and 7,090 did not. The duration of trials ranged from 4 weeks to 3 years, with median duration of 12 weeks. All published RCTs were deemed high quality studies based on the sequence generation, allocation concealment and double-blinding (Table 1b). At the outcome-level, RCTs were judged to be at high risk of bias because ascertainment of pneumonia was not performed systematically (Table 4). However, this bias would be non-differential as in blinded RCTs it would then similarly affect both intervention and control groups.

Table 4 Outcome-level quality assessment and summary of findings (GRADE)

\begin{tabular}{|c|c|c|c|c|c|c|c|c|c|c|c|}
\hline \multicolumn{12}{|c|}{ Pneumonia with ICS versus non-ICS } \\
\hline \multicolumn{7}{|c|}{ Quality assessment } & \multicolumn{5}{|c|}{ Summary of findings } \\
\hline \multirow[t]{2}{*}{$\begin{array}{l}\text { Participants } \\
\text { (studies) }\end{array}$} & \multirow[t]{2}{*}{$\begin{array}{l}\text { Risk } \\
\text { of bias }\end{array}$} & \multirow[t]{2}{*}{$\begin{array}{l}\text { Incon- } \\
\text { sistency }\end{array}$} & \multirow[t]{2}{*}{$\begin{array}{l}\text { Indirect- } \\
\text { ness }\end{array}$} & \multirow[t]{2}{*}{$\begin{array}{l}\text { Impre- } \\
\text { cision }\end{array}$} & \multirow{2}{*}{$\begin{array}{l}\text { Publica- } \\
\text { tion } \\
\text { bias }\end{array}$} & \multirow{2}{*}{$\begin{array}{l}\text { Overall } \\
\text { quality of } \\
\text { evidence }\end{array}$} & \multicolumn{2}{|c|}{$\begin{array}{l}\text { Study event rates } \\
\text { (\%) }\end{array}$} & \multirow{2}{*}{$\begin{array}{l}\text { Relative } \\
\text { effect } \\
(95 \% \mathrm{Cl})\end{array}$} & \multicolumn{2}{|c|}{$\begin{array}{l}\text { Anticipated } \\
\text { absolute effects }\end{array}$} \\
\hline & & & & & & & $\begin{array}{l}\text { Non- } \\
\text { ICS }\end{array}$ & ICS & & $\begin{array}{l}\text { Non- } \\
\text { ICS }\end{array}$ & $\begin{array}{l}\text { Risk with } \\
\text { ICS }(95 \% \mathrm{CI})\end{array}$ \\
\hline \multicolumn{12}{|l|}{ RCT } \\
\hline \multirow{4}{*}{$\begin{array}{l}19,098 \\
\text { (10 studies) }\end{array}$} & \multirow[t]{4}{*}{ High $^{1}$} & \multirow{4}{*}{$\begin{array}{l}\text { No } \\
\text { serious } \\
\text { incon- } \\
\text { sistency }\end{array}$} & \multirow{4}{*}{$\begin{array}{l}\text { No } \\
\text { serious } \\
\text { indirect- } \\
\text { ness }\end{array}$} & \multirow{4}{*}{$\begin{array}{l}\text { No } \\
\text { serious } \\
\text { impre- } \\
\text { cision }{ }^{2}\end{array}$} & \multirow{4}{*}{$\begin{array}{l}\text { Unde- } \\
\text { tected }\end{array}$} & \multirow{4}{*}{$\begin{array}{l}\bigoplus \oplus \oplus \ominus \\
\text { Moderate } \\
\text { due to risk } \\
\text { of bias }\end{array}$} & \multirow{4}{*}{$\begin{array}{l}128 / \\
7,090 \\
(1.8 \%)\end{array}$} & \multirow{4}{*}{$\begin{array}{l}116 / \\
12,008 \\
(1 \%)\end{array}$} & \multirow{4}{*}{$\begin{array}{l}\text { RR } 0.74 \\
(0.57 \text { to } \\
0.95)\end{array}$} & \multicolumn{2}{|c|}{ Study population } \\
\hline & & & & & & & & & & $\begin{array}{l}18 \\
\text { per } \\
1000\end{array}$ & $\begin{array}{l}5 \text { fewer } \\
\text { per } 1000 \\
\text { (from } 1 \\
\text { fewer to } 8 \\
\text { fewer) }\end{array}$ \\
\hline & & & & & & & & & & \multicolumn{2}{|c|}{ Moderate } \\
\hline & & & & & & & & & & $\begin{array}{l}3 \text { per } \\
1000\end{array}$ & $\begin{array}{l}1 \text { fewer per } \\
1000 \text { (from } \\
0 \text { fewer to } 1 \\
\text { fewer) }\end{array}$ \\
\hline \multicolumn{12}{|c|}{ Observational } \\
\hline \multirow{4}{*}{$\begin{array}{l}44,016 \\
\text { (4 studies) }\end{array}$} & \multirow{4}{*}{$\begin{array}{l}\text { Very } \\
\text { high }^{3,4,5}\end{array}$} & \multirow{4}{*}{$\begin{array}{l}\text { No } \\
\text { serious } \\
\text { incon- } \\
\text { sistency }\end{array}$} & \multirow{4}{*}{$\begin{array}{l}\text { No } \\
\text { serious } \\
\text { indirect- } \\
\text { ness }\end{array}$} & \multirow{4}{*}{$\begin{array}{l}\text { No } \\
\text { serious } \\
\text { impre- } \\
\text { cision }\end{array}$} & \multirow{4}{*}{$\begin{array}{l}\text { Unde- } \\
\text { tected }\end{array}$} & \multirow{4}{*}{$\begin{array}{l}\oplus \ominus \ominus \ominus \\
\text { Very low }{ }^{3,4,5} \\
\text { due to risk } \\
\text { of bias }\end{array}$} & \multirow{4}{*}{$\begin{array}{l}3,733 / \\
28,213 \\
(13.3 \%)\end{array}$} & \multirow{4}{*}{$\begin{array}{l}3,517 / \\
15,803 \\
(22.3 \%)\end{array}$} & & Study & opulation \\
\hline & & & & & & & & & $\begin{array}{l}(1.87 \text { to } \\
2.07)\end{array}$ & $\begin{array}{l}133 \\
\text { per } \\
1000\end{array}$ & $\begin{array}{l}99 \text { more } \\
\text { per } 1000 \\
\text { (from } 90 \\
\text { more to } \\
108 \text { more) }\end{array}$ \\
\hline & & & & & & & & & & Mode & ate \\
\hline & & & & & & & & & & $\begin{array}{l}333 \\
\text { per } \\
1000\end{array}$ & $\begin{array}{l}163 \text { more } \\
\text { per } 1000 \\
\text { (from } 150 \\
\text { more to } \\
175 \text { more) }\end{array}$ \\
\hline
\end{tabular}

'Limited pneumonia ascertainment; ${ }^{2}$ Although several trials had wide confidence intervals, these represented less than $5 \%$ of the weight; ${ }^{3} \mathrm{Case}$ control and historical cohort designs; ${ }^{4}$ Unaccounted step up in ICS therapy due to persistent respiratory symptoms preceding the diagnosis of pneumonia; ${ }^{5}$ One study carried $98 \%$ of overall weight. 
The estimated overall unadjusted risk of pneumonia with the use of ICS in RCTs, was in protective range; $\mathrm{RR} 0.74,95 \% \mathrm{CI} 0.57$ to $0.95, \mathrm{p}=0.02$, without any heterogeneity (Figure 2). As the details on 26 unpub- lished RCTs reported in the single study by O'Byrne et al. (21) were not available, we performed a sensitivity analysis. When we excluded results of O'Byrne study (21), the confidence interval extended to $1(95 \% \mathrm{CI}$

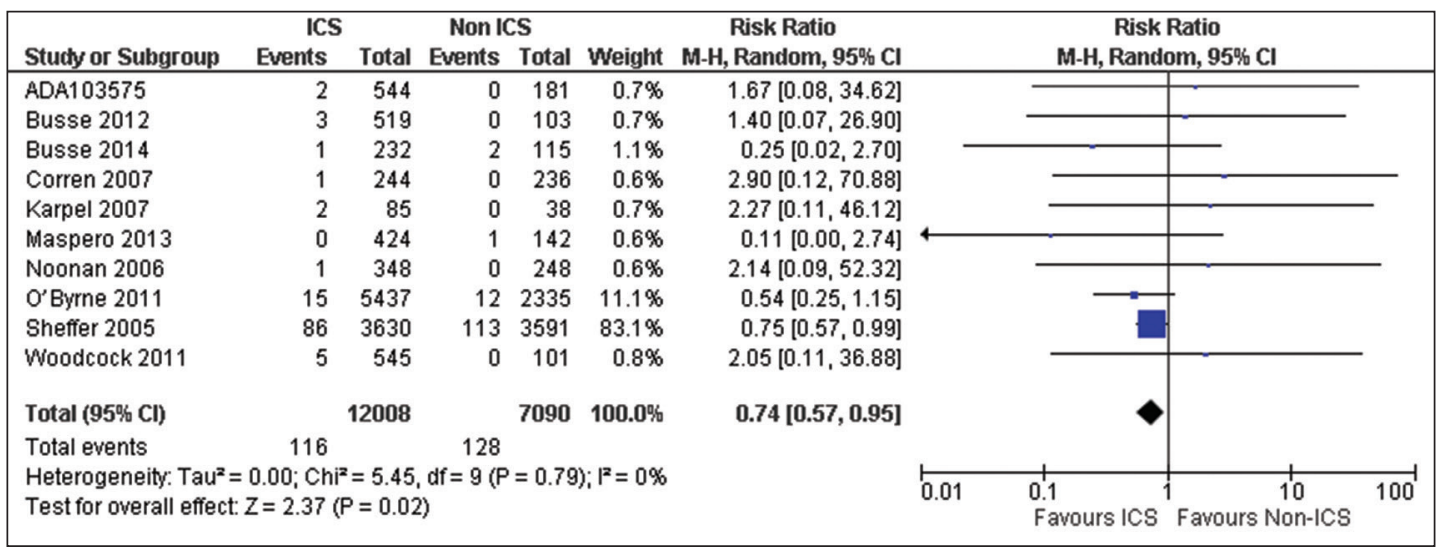

Figure 2 Meta-analysis of RCTs for incident pneumonia.

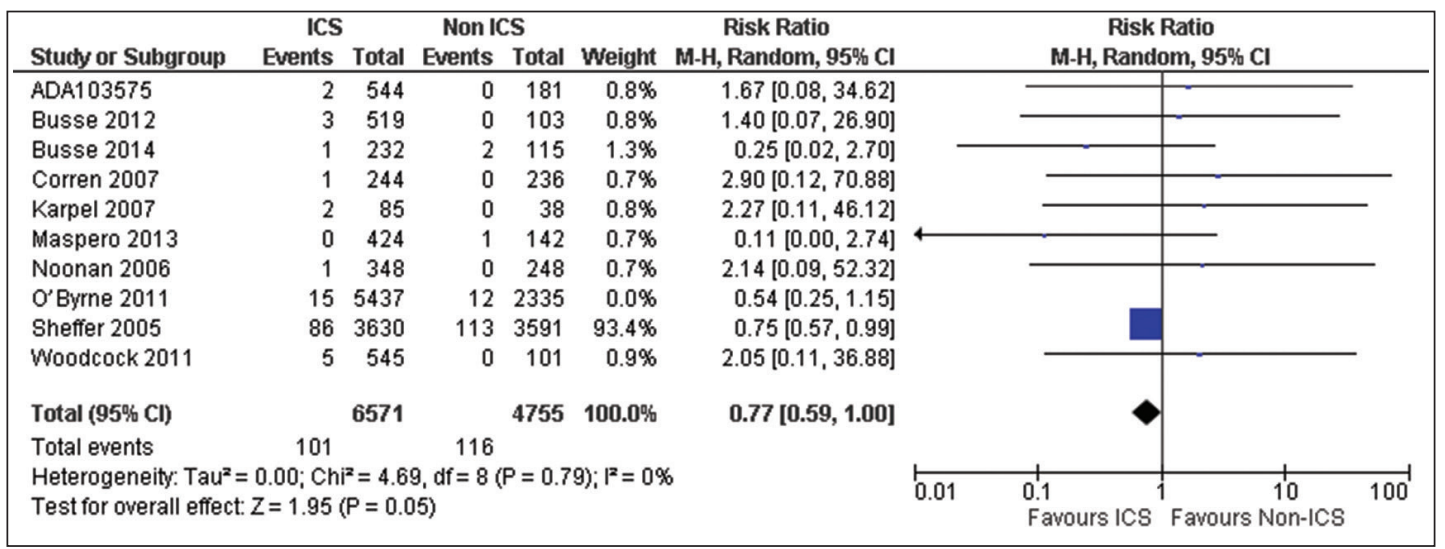

Figure 3 Sensitivity analyses of RCT data - A) Without O’Byrne 2011 study data.

\begin{tabular}{|c|c|c|c|c|c|c|c|c|c|c|}
\hline \multirow[b]{2}{*}{ Study or Subgroup } & \multicolumn{2}{|c|}{ ICS } & \multicolumn{2}{|c|}{ Non ICS } & \multirow[b]{2}{*}{ Weight } & \multirow{2}{*}{$\begin{array}{c}\text { Risk Ratio } \\
\text { M-H, Random, 95\% Cl }\end{array}$} & \multirow{2}{*}{\multicolumn{4}{|c|}{$\begin{array}{c}\text { Risk Ratio } \\
\text { M-H, Random, 95\% Cl }\end{array}$}} \\
\hline & Events & Total & Events & Total & & & & & & \\
\hline ADA103575 & 2 & 544 & 0 & 181 & $4.1 \%$ & $1.67[0.08,34.62]$ & & & & \\
\hline Busse 2012 & 3 & 519 & 0 & 103 & $4.3 \%$ & $1.40[0.07,26.90]$ & & & & \\
\hline Corren 2007 & 1 & 244 & 0 & 236 & $3.7 \%$ & $2.90[0.12,70.88]$ & & & & \\
\hline Karpel 2007 & 2 & 85 & 0 & 38 & $4.1 \%$ & $2.27[0.11,46.12]$ & & & & \\
\hline Maspero 2013 & 0 & 424 & 1 & 142 & $3.7 \%$ & $0.11[0.00,2.74]$ & & & & \\
\hline Sheffer 2005 & 86 & 3630 & 113 & 3591 & $0.0 \%$ & $0.75[0.57,0.99]$ & & & & \\
\hline Woodcock 2011 & 5 & 545 & 0 & 101 & $4.5 \%$ & $2.05[0.11,36.88]$ & & & & \\
\hline Total $(95 \% \mathrm{Cl})$ & & 8378 & & 3499 & $100.0 \%$ & $0.66[0.36,1.22]$ & & & & \\
\hline Total events & 30 & & 15 & & & & & & & \\
\hline
\end{tabular}

Figure 3 Sensitivity analyses of RCT data - B) Without Sheffer 2005 study data. 


\begin{tabular}{|c|c|c|c|c|c|c|c|c|c|c|}
\hline Study or Subgroup & \multicolumn{2}{|l|}{ ICS } & \multicolumn{2}{|c|}{ Non ICS } & Weight & $\begin{array}{c}\text { Risk Ratio } \\
\text { M-H, Random, } 95 \% \mathrm{Cl}\end{array}$ & \multicolumn{4}{|c|}{$\begin{array}{c}\text { Risk Ratio } \\
\text { M-H, Random, } 95 \% \text { Cl }\end{array}$} \\
\hline ADA103575 & 406 & 544 & 138 & 181 & $12.2 \%$ & $0.98[0.89,1.08]$ & & & & \\
\hline Busse 2012 & 450 & 519 & 65 & 103 & $11.1 \%$ & $1.37[1.18,1.60]$ & & & - & \\
\hline Busse 2014 & 186 & 232 & 77 & 115 & $11.3 \%$ & $1.20[1.04,1.38]$ & & & $=$ & \\
\hline Corren 2007 & 208 & 244 & 139 & 236 & $11.8 \%$ & $1.45[1.29,1.63]$ & & &. & \\
\hline Karpel 2007 & 71 & 85 & 16 & 38 & $6.2 \%$ & $1.98[1.35,2.91]$ & & & $\rightarrow$ & \\
\hline Maspero 2013 & 317 & 424 & 111 & 142 & $12.1 \%$ & $0.96[0.86,1.06]$ & & & & \\
\hline Noonan 2006 & 261 & 348 & 110 & 248 & $11.1 \%$ & $1.69[1.45,1.97]$ & & & $=$ & \\
\hline Sheffer 2005 & 2640 & 3630 & 2571 & 3591 & $13.0 \%$ & $1.02[0.99,1.05]$ & & & & \\
\hline Woodcock 2011 & 455 & 545 & 65 & 101 & $11.2 \%$ & $1.30[1.12,1.51]$ & & & - & \\
\hline Total $(95 \% \mathrm{Cl})$ & & 6571 & & 4755 & $100.0 \%$ & $1.25[1.10,1.43]$ & & & $\checkmark$ & \\
\hline Total events & 4994 & & 3292 & & & & & & & \\
\hline $\begin{array}{l}\text { Heterogeneity: Tau }{ }^{2} \\
\text { Test for overall effect }\end{array}$ & $\begin{array}{l}0.03 ; \mathrm{Chi}^{-} \\
\mathrm{z}=3.32(\end{array}$ & $\begin{array}{l}F^{2}=111 \\
P=0.0\end{array}$ & $\begin{array}{l}.52, \mathrm{df}= \\
0009)\end{array}$ & $8(P<C$ & 0.00001); & $F^{2}=93 \%$ & 0.01 & $\begin{array}{l}0.1 \\
\text { Favours ICS }\end{array}$ & $1 \frac{10}{1} \quad \frac{1}{\text { Favours Non-ICS }}$ & 100 \\
\hline
\end{tabular}

Figure 4 Study completion rates comparing ICS and non-ICS groups.

0.59-to $1, \mathrm{p}=0.05$ ) (Figure $3 \mathrm{~A}$ ). As a result, the weight of study by Sheffer et al. (30) in the meta-analysis consequently increased from $83.1 \%$ to $93.4 \%$. This study was one of the three published START trial reports (30, $38,39)$. Once the study by Sheffer et al. was removed because of the overly dominant weight, in a subsequent sensitivity analysis the pre-hospital use of ICS in asthma patients did not show significant protective effect for pneumonia any longer (RR 0.66, 95\% CI 0.36 to $1.22, \mathrm{p}=0.19$ ) possibly due to loss of power, as $80 \%$ of pneumonia cases were consequently excluded from the analysis (Figure $3 \mathrm{~B}$ ).

We also assessed the study completion rates between the ICS and non-ICS groups in the RCTs. Eight RCTs were estimable and the trial completion rate was higher in the non-ICS than in the ICS group; RR 1.25; 95\% CI 1.10to 1.43, $\mathrm{p}=<0.001 ; \mathrm{I}^{2}=93 \%$ (Figure 4). Only 2 RCTs reported occurrence of deaths; there were total of 13 deaths, 5 in ICS and 8 in non-ICS group, respectively $(30,33)$.

\section{Observational studies}

We initially included 8 observational studies $(19,20,22,23,40-43)$. Five cohort studies $(19,22,40,42,43)$ excluded patients on systemic corticosteroids and three case-control studies $(20,23,41)$ adjusted for systemic corticosteroid use. Two studies assessed risk of outpatient pneumonia $(20,23)$, five assessed pneumonias requiring admission to the hospital $(19,22,40,42,43)$, and one study (41) assessed risk of non-tuberculous mycobacteriosis by using NTM index rate. Although ascertainment of pneumonia in observational studies was more systematic by using not only clinical diagnosis but radiographic assessment as well, all observational studies were judged to be at very high risk of bias (Tables 2 and 4). Four observational studies were not estimable for unadjusted risk of pneumonia as they included only patients with pneumonia so the unadjusted differential risk of ICS could not be estimated. The remaining 4 estimable studies included 44,016 patients, of whom 15,803 were on ICS and 28,213 were not on ICS. The risk of incident pneumonia was found to be increased; OR 1.97; 95\% CI 1.87to 2.07, $\mathrm{p}<0.0001$, with no observed heterogeneity (Figure 5). Three studies were case-control studies and one was secondary analysis of a large cohort (Table 2). Of note, recently published study by McKeever et al. (23) carried almost complete weight (98\%) in this meta-analysis. Once this study was excluded in a sensitivity analysis (Figure 6), the estimated effect decreased appreciably to OR 1.57; 95\% CI 1.09 to 2.25). Exclusion of a single study that assessed risk of NTM (41) did not change the results significantly. 


\begin{tabular}{|c|c|c|c|c|c|c|c|c|c|c|}
\hline \multirow[b]{2}{*}{ Study or Subgroup } & \multicolumn{2}{|c|}{ ICS } & \multicolumn{2}{|c|}{ Non ICS } & \multirow[b]{2}{*}{ Weight } & \multirow{2}{*}{$\begin{array}{c}\text { Odds Ratio } \\
\text { M-H, Random, } 95 \% \mathrm{Cl}\end{array}$} & & \multirow{2}{*}{\multicolumn{2}{|c|}{$\begin{array}{c}\text { Odds Ratio } \\
\text { M-H, Random, } 95 \% \mathrm{Cl}\end{array}$}} & \\
\hline & Events & Total & Events & Total & & & & & & \\
\hline Almirall 2010 & 22 & 30 & 210 & 344 & $0.4 \%$ & $1.75[0.76,4.06]$ & & & & \\
\hline Andrejak 2013 & 9 & 30 & 1 & 3 & $0.0 \%$ & $0.86[0.07,10.70]$ & & & & \\
\hline Festic 2014 & 61 & 149 & 90 & 291 & $1.6 \%$ & $1.55[1.03,2.33]$ & & & - & \\
\hline Mckeever 2013 & 3425 & 15594 & 3432 & 27575 & $98.0 \%$ & $1.98[1.88,2.09]$ & & & & \\
\hline Total (95\% Cl) & & 15803 & & 28213 & $100.0 \%$ & $1.97[1.87,2.07]$ & & & 1 & \\
\hline Total events & 3517 & & 3733 & & & & & & & \\
\hline $\begin{array}{l}\text { Heterogeneity: Tau } \\
\text { Test for overall effec }\end{array}$ & $\begin{array}{l}0.00 ; \mathrm{Chi} \\
\mathrm{Z}=25.77\end{array}$ & $\begin{array}{l}2=1.85 \\
(P<0.0\end{array}$ & $\begin{array}{l}d f=3(P \\
0001)\end{array}$ & $=0.60) ;$ & $1^{2}=0 \%$ & & 0.01 & ${ }_{\text {Favours ICS }}^{0.1}$ & $\begin{array}{l}10 \\
\text { Favours Non-ICS }\end{array}$ & 100 \\
\hline
\end{tabular}

Figure 5 Meta-analysis of observational studies for incident pneumonia.

\begin{tabular}{|c|c|c|c|c|c|c|c|c|c|c|}
\hline \multirow[b]{2}{*}{ Study or Subgroup } & \multicolumn{2}{|c|}{ ICS } & \multicolumn{2}{|c|}{ Non ICS } & \multirow[b]{2}{*}{ Weight } & \multirow{2}{*}{$\begin{array}{c}\text { Odds Ratio } \\
\text { M-H, Random, } 95 \% \mathrm{Cl}\end{array}$} & \multirow{2}{*}{\multicolumn{4}{|c|}{$\begin{array}{c}\text { Odds Ratio } \\
\text { M-H, Random, } 95 \% \mathrm{Cl}\end{array}$}} \\
\hline & Events & Total & Events & Total & & & & & & \\
\hline Almirall 2010 & 22 & 30 & 210 & 344 & $19.0 \%$ & $1.75[0.76,4.06]$ & & & & \\
\hline Andrejak 2013 & 9 & 30 & 1 & 3 & $2.1 \%$ & $0.86[0.07,10.70]$ & & & & \\
\hline Festic 2014 & 61 & 149 & 90 & 291 & $79.0 \%$ & $1.55[1.03,2.33]$ & & & & \\
\hline Mckeever 2013 & 3425 & 15594 & 3432 & 27575 & $0.0 \%$ & $1.98[1.88,2.09]$ & & & & \\
\hline Total $(95 \% \mathrm{Cl})$ & & 209 & & 638 & $100.0 \%$ & $1.57[1.09,2.25]$ & & & & \\
\hline \multirow{2}{*}{\multicolumn{6}{|c|}{$\begin{array}{l}\text { Total events } \quad 92 \quad 301 \\
\text { Heterogeneity: } \mathrm{Tau}^{2}=0.00 ; \mathrm{Chi}^{2}=0.29, \mathrm{df}=2(\mathrm{P}=0.86) ; \mathrm{l}^{2}=0 \% \\
\text { Test for overall effect: } Z=2.41(P=0.02)\end{array}$}} & & & & & \\
\hline & & & & & & & 0.01 & $\begin{array}{l}0.1 \\
\text { Favours ICS }\end{array}$ & $\begin{array}{c}10 \\
\text { Favours Non-ICS }\end{array}$ & 100 \\
\hline
\end{tabular}

Figure 6 Sensitivity analysis for observational studies.

Mortality was reported only in two observational studies $(20,43) ; 2$ in ICS and 6 in non-ICS group. The observed low mortality rates in both RCTs and observational studies precluded performance of pooled analysis.

\section{Discussion}

Based on available RCTs, this meta-analysis suggests that ICS are associated with decreased risk of incident pneumonia in asthma patients for the duration of respective clinical trials. Although observational studies suggested increased risk of incident pneumonia in similar patients using ICS, the inherent methodological limitations and higher risk of bias conferred lower grade of confidence in the findings of the observational studies. To our knowledge, this is the first systematic review and meta-analysis of all available RCTs and observational studies assessing the association between ICS use and risk of incident pneumonia in asthma patients.

There has been a clinical controversy regarding the risk of pneumonia in patients on ICS with COPD and asthma. Since the TORCH study reported increased incidence of pneumonia among COPD patients in 2007 (14), several well-designed trials and meta-analyses demonstrated the similar risk (44-49). However, the risk for developing community acquired pneumonia among asthma patients on ICS did not appear to be substantially increased (18-22).

Our meta-analysis of RCTs suggests that ICS are associated with decreased risk of incident pneumonia in asthmatic patients. There was no heterogeneity and there was overall less risk of bias compared to observational studies. It is uncertain why the use of ICS may be associated with an increased risk of incident pneumonia in patients with COPD but not asthma. It has been hypothesized that ICS more efficiently reduce airway inflammation, segmental atelectasis, mucoid impaction, and thus, subsequent pneumonia in patients with asthma compared with those with COPD (50). Additionally, patients with COPD are commonly of older age and have a greater burden of comorbid diseases than asthmatics, which are recognized risk fac- 
tors for pneumonia. Thus, higher observed pneumonia rates in COPD patients compared to patients with asthma may be partly explained by difference in age and comorbidities. Importantly, two RCTs $(29,34)$ in asthma patients were shorter than 12 weeks, while the shortest clinical trials in COPD patients were 24-week long. It is conceivable that any proposed medication adverse effect could become more apparent in the studies of longer duration. Moreover, we observed higher study completion rate among non-ICS patients compared to ICS patients in eight estimable RCTs in asthma patients, however with very high heterogeneity.

We believe that it is safer to conclude that the incident risk of pneumonia with the ICS use in RCTs of asthma patients was not increased, rather than it was decreased. Although the primary meta-analysis of RCTs suggested that ICS might carry protective effect, once we excluded the trial by Sheffer et al. (30) in a sensitivity analysis, the protective effect was not statistically significant any longer. A possible explanation for the "overinflated" protective effect observed in this particular study could have been incorrect allocation of respiratory events known to be improved by ICS as pneumonia adverse events. These events could have been: segmental atelectasis due to mucous impaction that is more often seen in children with poorly controlled asthma; increased cough and mucous production; or mild asthma exacerbations. This interpretation is supported by the fact that the most frequent reporting of pneumonia adverse events was in children aged 5 to 11 years, in whom atelectasis is more frequently seen as a consequence of asthma exacerbation than in adult patients (21). Since ICS effectively improve flow limitation in asthma patients, the patients on ICS could have had less incorrectly allocated respiratory events as pneumonia. Moreover, study by Sheffer et al. included only preparations of budesonide, which has been shown previously to have more rapid clearance from the airways and to be less potent than fluticasone $(51,52)$. However, this is only speculative and would require future well designed prospective studies with a strict definition for pneumonia to fully resolve.

On the contrary, the pooling of observational studies suggested higher risk of pneumonia in patients with asthma. A single study by McKeever et al. carried almost complete weight in this meta-analysis (23). This recently published case-control study showed that asthma patients admitted with pneumonia were more likely to have prescription for ICS than the control subjects in the preceding 90 days; they were also more likely to use reliever inhalers and oral steroids in the previous year. The investigators used clinical diagnosis of pneumonia and did not necessarily base it on the findings on chest radiographs. Therefore, we propose that a substantial number of patients who were retrospectively included in this study (and other similar observational studies) may have had unrecognized pneumonia leading to persistent respiratory symptoms prompting increased asthma therapy containing ICS. The authors recognize this limitation of their study (23) and concluded that the prescribers should consider possibility of incipient infection rather than underlying asthma being responsible for the worsening respiratory symptoms before prescribing or increasing ICS dose. The similar was also previously demonstrated in a study on COPD patients by Calverley et al. (47). The data interpretation from this study's daily record cards suggested identical numbers of de novo pneumonias in both ICS and non-ICS arms, but more unresolved exacerbations preceding pneumonia events in the ICS-treated COPD patients. Finally, it is possible that some patients with concomitant COPD were included in the observational studies of asthma patients, which was likely not the case in RCTs. 
Although the overall pneumonia ascertainment was more systematic in most of the observational studies compared to RCTs, the resulting overall grade of confidence was lower for observational studies compared to the randomized trials due to very high risk of bias (mainly indication and selection bias) (Table 4). Of note, using GRADE profiler for outcome-level quality assessment may be associated with the rater-dependent subjectivity. After cautiously analyzing and weighing all available pertinent factors, we proposed "moderate" and "very low" quality grades for analyzed RCTs and observational studies, respectively. The limitation of RCTs lacking systematic ascertainment of pneumonia would be an example of nondifferential bias; therefore we labeled this as "high" rather than "very high" risk of bias. However, even if RCTs were downgraded to "low" quality given concerns with pneumonia ascertainment, the overall resulting grades of confidence would still favor RCTs rather than observational studies on the topic, which were not necessarily populationbased observational studies.

Our meta-analysis has several limitations, some of which are attributable to methodological shortcomings in the studies included. We were somewhat surprised with the relatively small number of studies retrieved by our search. The reason for this could be that either study investigators did not systematically measure incident pneumonia events (including radiographic assessment), or less likely they did not report those in their publications. Therefore, our review is prone to the reporting bias as we depended solely on the reporting of outcomes. There is also publication bias risk as all RCT were pharmaceutical industryfunded. However, we reviewed the clinical trials registry and included both published and unpublished studies. The resulting funnel plot of RCTs did not suggest publication bias (Supplemental material). Although the risk of pneumonia was unadjusted, the large number of patients included in the metaanalysis partially alleviated this concern. We did not have individual-patient data, so we could not detect any differences in pneumonia based on demographics, asthma severity or presence of comorbidities. The time of follow-up in included studies differed widely, which may have also impacted results. We considered all patients on ICS as ICS users regardless of ICS being used alone or in combination with another medication. Also, non-ICS users were considered all patients not on ICS regardless of use of additional medications, such as long-acting beta agonists or placebo. This is justified by our stated main intention of assessment for the overall association of ICS and pneumonia. Only future prospective trials of ICS designed to systematically assess and monitor pneumonia as a pre-specified outcome using an objective pneumonia definition could alleviate the above-mentioned limitations.

\section{Conclusion}

Results from our meta-analysis on available RCTs suggest that ICS use in patients with asthma was associated with decreased risk of pneumonia. On the contrary, a metaanalysis of observational studies suggested a higher risk of pneumonia in similar patients; however, the grade of confidence in this subgroup's results is lower due to inherent methodological limitations. The design of future prospective trials of ICS should include systematic assessment and monitoring of pneumonia as a pre-specified outcome.

What is already known on this subject

Inhaled corticosteroids are the mainstay of asthma treatment for all ages. Their use has been previously associated with increased risk of pneumonia among COPD patients. It is uncertain if there is an association between long-term use of inhaled corticosteroids and the incident pneumonia among asthmatic patients. 


\section{What this study adds}

This is the first systematic review on use of inhaled corticosteroids and incident pneumonia in asthmatic patients. It includes randomized clinical trials as well as observational studies, which were pooled in the two separate meta-analyses. While randomized clinical trials showed decreased risk of incident pneumonia, this risk was increased in observational studies, which were at higher risk of bias and conferred lower grade of confidence.

\section{Acknowledgements:}

1. All authors contributed substantially to the study design, data analysis and interpretation, and the writing of the manuscript. They all had full access to all of the data in the study and take responsibility for the integrity of the data and the accuracy of the data analysis.

2. We acknowledge Patricia Erwin, the head reference librarian at Mayo Clinic, Rochester, MN for her help with the library search.

3. We acknowledge Dr. P. J. Almirall and Dr. Miquel Ferrer for their correspondence and contribution to our manuscript.

4. The abstract was presented as an oral presentation at the last Annual Scientific Meeting of American College of Allergy, Asthma \& Immunology, in Atlanta, GA.

Authors' contributions: Conception and design: EF, VB, MAM and MMJ; Acquisition analysis and interpretation of data: EF, VB, MAM and MMJ; Drafting the article: EF, VB, MAM and MMJ; Revising it critically for important intellectual content: EF, VB, MAM and MMJ.

Conflict of interest: The authors declare that they have no conflict of interest.

Funding: Supported in part by grants from the National Center for Advancing Translational Sciences (grant no. 5KL2TR000136-08 and grant no. CTSA UL1 TR000135), a component of the National Institutes of Health (NIH) and Mayo Foundation. The views expressed in this article do not communicate an official position of the NIH and Mayo Foundation.

\section{References}

1. Barnes PJ, Pedersen S. Efficacy and safety of inhaled corticosteroids in asthma. Report of a workshop held in Eze, France, October 1992. Am Rev Respir Dis. 1993;148(4 Pt 2):S1-26.

2. Ernst P, Suissa S. Systemic effects of inhaled corticosteroids. Curr Opin Pulm Med. 2012;18(1):85-9.
3. Juniper EF, Kline PA, Vanzieleghem MA, Ramsdale EH, O’Byrne PM, Hargreave FE. Effect of long-term treatment with an inhaled corticosteroid (budesonide) on airway hyperresponsiveness and clinical asthma in nonsteroid-dependent asthmatics. Am Rev Respir Dis. 1990;142(4):832-6.

4. Pauwels RA, Löfdahl CG, Postma DS, Tattersfield AE, O’Byrne P, Barnes PJ, et al. Effect of inhaled formoterol and budesonide on exacerbations of asthma. Formoterol and Corticosteroids Establishing Therapy (FACET) International Study Group. N Engl J Med. 1997;337(20):1405-11.

5. Suissa S, Ernst P. Inhaled corticosteroids: impact on asthma morbidity and mortality. J Allergy Clin Immunol. 2001;107(6):937-44.

6. Suissa S, Ernst P, Benayoun S, Baltzan M, Cai B. Low-dose inhaled corticosteroids and the prevention of death from asthma. N Engl J Med. 2000;343(5):332-6.

7. O’Byrne PM, Barnes PJ, Rodriguez-Roisin R, Runnerstrom E, Sandstrom T, Svensson K, et al. Low dose inhaled budesonide and formoterol in mild persistent asthma: the OPTIMA randomized trial. Am J Respir Crit Care Med. 2001;164(8 Pt 1):1392-7.

8. Bateman ED, Boushey HA, Bousquet J, Busse WW, Clark TJ, Pauwels RA, et al. Can guidelinedefined asthma control be achieved? The Gaining Optimal Asthma ControL study. Am J Respir Crit Care Med. 2004;170(8):836-44.

9. Cumming RG, Mitchell P, Leeder SR. Use of inhaled corticosteroids and the risk of cataracts. $\mathrm{N}$ Engl J Med. 1997;337(1):8-14.

10. Uboweja A, Malhotra S, Pandhi P. Effect of inhaled corticosteroids on risk of development of cataract: a meta-analysis. Fundam Clin Pharmacol. 2006;20(3):305-9.

11. Slatore CG, Bryson CL, Au DH. The association of inhaled corticosteroid use with serum glucose concentration in a large cohort. Am J Med. 2009;122(5):472-8.

12. Suissa S, Kezouh A, Ernst P. Inhaled corticosteroids and the risks of diabetes onset and progression. Am J Med. 2010;123(11):1001-6.

13. O’Byrne PM, Rennard S, Gerstein H, Radnerc F, Petersonc S, Lindberget B, et al. Risk of new onset diabetes mellitus in patients with asthma or COPD taking inhaled corticosteroids. Respir Med. 2012;106(11):1487-93.

14. Calverley PM, Anderson JA, Celli B, Ferguson GT, Jenkins C, Jones PW, et al. Salmeterol and fluticasone propionate and survival in chronic obstructive pulmonary disease. N Engl J Med. 2007;356(8):775-89. 
15. Drummond MB, Dasenbrook EC, Pitz MW, Murphy DJ, Fan E. Inhaled corticosteroids in patients with stable chronic obstructive pulmonary disease: a systematic review and meta-analysis. JAMA. 2008;300(20):2407-16.

16. Rodrigo GJ, Castro-Rodriguez JA, Plaza V. Safety and efficacy of combined long-acting beta-agonists and inhaled corticosteroids vs long-acting beta-agonists monotherapy for stable COPD: a systematic review. Chest. 2009;136(4):1029-38.

17. Joo MJ, Au DH, Fitzgibbon ML, Lee TA. Inhaled corticosteroids and risk of pneumonia in newly diagnosed COPD. Respir Med. 2010;104(2):24652.

18. Kobayashi N, Lisura M. Bacterial pneumonia in asthmatic patients. Arerugika. 2002;13:329-35.

19. To M, To Y, Yamada H, Ogawa C, Otomo M, Suzuki N, et al. Influence of inhaled corticosteroids on community-acquired pneumonia in patients with bronchial asthma. Intern Med. 2004;43(8):674-8.

20. Almirall J, Bolíbar I, Serra-Prat M, Palomera E, Roig J, Hospital I, et al. Inhaled drugs as risk factors for community-acquired pneumonia. Eur Respir J. 2010;36(5):1080-7.

21. O'Byrne PM, Pedersen S, Carlsson LG, Radner F, Thorén A, Peterson S, et al. Risks of pneumonia in patients with asthma taking inhaled corticosteroids. Am J Respir Crit Care Med. 2011;183(5):589-95.

22. Festic E, Bansal V, Gajic O, Lee AS. Prehospital use of inhaled corticosteroids and point prevalence of pneumonia at the time of hospital admission: secondary analysis of a multicenter cohort study. Mayo Clin Proc. 2014;89(2):154-62.

23. McKeever T, Harrison TW, Hubbard R, Shaw D. Inhaled corticosteroids and the risk of pneumonia in people with asthma: a case-control study. Chest. 2013;144(6):1788-94.

24. Koivula I, Sten M, Makela PH. Risk factors for pneumonia in the elderly. Am J Med. 1994;96(4):313-20.

25. Talbot TR, Hartert TV, Mitchel E, Halasa NB, Arbogast PG, Poehling KA, et al. Asthma as a risk factor for invasive pneumococcal disease. N Engl J Med. 2005;352(20):2082-90.

26. Almirall J, Bolíbar I, Serra-Prat M, Roig J, Hospital I, Carandell E, et al. New evidence of risk factors for community-acquired pneumonia: a population-based study. Eur Respir J. 2008;31(6):127484.

27. Juhn YJ, Kita H, Yawn BP, Boyce TG, Yoo KH, McGree ME, et al. Increased risk of serious pneumococcal disease in patients with asthma. J Allergy Clin Immunol. 2008;122(4):719-23.
28. Klemets P, Lyytikäinen O, Ruutu P, Ollgren J, Kaijalainen T, Leinonen $M$, et al. Risk of invasive pneumococcal infections among working age adults with asthma. Thorax. 2010;65(8):698-702.

29. Study No. ADA103575 Clinicaltrials.gov Identifier NCT00296491. [cited 2014 June 17]. Available from: http://www.gsk-clinicalstudyregister.com/ study/ADA103575?study_ids=ADA103575\#ps.

30. Sheffer AL, Silverman M, Woolcock AJ, Diaz PV, Lindberg B, Lindmark B. Long-term safety of once-daily budesonide in patients with early-onset mild persistent asthma: results of the Inhaled Steroid Treatment as Regular Therapy in Early Asthma (START) study. Ann Allergy Asthma Immunol. 2005;94(1):48-54.

31. Noonan M, Rosenwasser LJ, Martin P, O'Brien CD, O'Dowd L. Efficacy and safety of budesonide and formoterol in one pressurised metered-dose inhaler in adults and adolescents with moderate to severe asthma: a randomised clinical trial. Drugs. 2006;66(17):2235-54.

32. Corren J, Korenblat PE, Miller CJ, O’Brien CD, Mezzanotte WS. Twelve-week, randomized, placebo-controlled, multicenter study of the efficacy and tolerability of budesonide and formoterol in one metered-dose inhaler compared with budesonide alone and formoterol alone in adolescents and adults with asthma. Clin Ther. 2007;29(5):823-43.

33. Karpel JP, Nayak A, Lumry W, Craig TJ, Kerwin E, Fish JE, et al. Inhaled mometasone furoate reduces oral prednisone usage and improves lung function in severe persistent asthma. Respir Med. 2007;101(3):628-37.

34. Woodcock A, Bateman ED, Busse WW, Lötvall J, Snowise NG, Forth R, et al. Efficacy in asthma of once-daily treatment with fluticasone furoate: a randomized, placebo-controlled trial. Respir Res. 2011;12:132.

35. Busse WW, Bleecker ER, Bateman ED, Lötvall J, Forth R, Davis AM, et al. Fluticasone furoate demonstrates efficacy in patients with asthma symptomatic on medium doses of inhaled corticosteroid therapy: an 8-week, randomised, placebocontrolled trial. Thorax. 2012;67(1):35-41.

36. Maspero J, Backer V, Yao R, Staudinger H, Teper A. Effects of mometasone, fluticasone, and montelukast on bone mineral density in adults with asthma. J Allergy Clin Immunol Pract. 2013;1(6):64955.e1.

37. Busse WW, Bateman ED, O’Byrne PM, Lötvall J, Woodcock A, Medley H, et al. Once-daily fluticasone furoate $50 \mathrm{mcg}$ in mild-to-moderate asthma: a 24-week placebo-controlled randomized trial. Allergy. 2014;69(11):1522-30. 
38. Pauwels RA, Pedersen S, Busse WW, Tan WC, Chen YZ, Ohlsson SV, et al. Early intervention with budesonide in mild persistent asthma: a randomised, double-blind trial. Lancet. 2003;361(9363):1071-6.

39. O'Byrne PM, Pedersen S, Lamm CJ, Tan WC, Busse WW. Severe exacerbations and decline in lung function in asthma. Am J Respir Crit Care Med. 2009;179(1):19-24.

40. Sellares J, López-Giraldo A, Lucena C, Cilloniz C, Amaro R, Polverino E, et al. Influence of previous use of inhaled corticoids on the development of pleural effusion in community-acquired pneumonia. Am J Respir Crit Care Med. 2013;187(11):1241-8.

41. Andrejak C, Nielsen R, Thomsen VO, Duhaut P, Sorensen HT, Thomsen RW. Chronic respiratory disease, inhaled corticosteroids and risk of non-tuberculous mycobacteriosis. Thorax. 2013;68(3):256-62.

42. Ferrer M, Torres A, Martínez R, Ramírez P, Polverino E, Montull B, et al. Inhaled corticosteroids and systemic inflammatory response in community-acquired pneumonia: a prospective clinical study. Respirology. 2014;19(6):929-35.

43. Terraneo S, Polverino E, Cilloniz C, Amaro R, Vennera Mdel C, Gabarrus A, et al. Severity and outcomes of community acquired pneumonia in asthmatic patients. Respir Med. 2014;108(11):1713-22.

44. Ferguson GT, Anzueto A, Fei R, Emmett A, Knobil K, Kalberg C. Effect of fluticasone propionate/ salmeterol (250/50 microg) or salmeterol (50 microg) on COPD exacerbations. Respir Med. 2008;102(8):1099-108.

45. Wedzicha JA, Calverley PMA, Seemungal TA, Hagan G, Ansari Z, Stockley RA. The prevention of chronic obstructive pulmonary disease exacerbations by salmeterol/fluticasone propionate or tiotropium bromide. Am J Respir Crit Care Med. 2008;177(1):19-26.

46. Anzueto A, Ferguson GT, Feldman G, Chinsky K, Seibert A, Emmett A, et al. Effect of fluticasone propionate/salmeterol (250/50) on COPD exacerbations and impact on patient outcomes. COPD. 2009;6(5):320-9.

47. Calverley PM, Stockley RA, Seemungal TA, Hagan G, Willits LR, Riley JH, et al. Reported pneumonia in patients with COPD: findings from the INSPIRE study. Chest. 2011;139(3):505-12.

48. Sharafkhaneh A, Southard JG, Goldman M, Uryniak T, Martin UJ. Effect of budesonide/formoterol pMDI on COPD exacerbations: a double-blind, randomized study. Respir Med. 2012;106(2):25768.

49. Dransfield MT, Bourbeau J, Jones PW, Hanania NA, Mahler DA, Vestbo J, et al. Once-daily inhaled fluticasone furoate and vilanterol versus vilanterol only for prevention of exacerbations of COPD: two replicate double-blind, parallel-group, randomised controlled trials. Lancet Respir Med. 2013;1(3):210-23.

50. Schleimer RP. An overview of glucocorticoid anti-inflammatory actions. Eur J Clin Pharmacol. 1993;45 Suppl 1:S3-7; discussion S43-4.

51. Boorsma M, Andersson N, Larsson P, Ullman A. Assessment of the relative systemic potency of inhaled fluticasone and budesonide. Eur Respir J. 1996;9(7):1427-32.

52. Thorsson L, Edsbäcker S, Källén A, Löfdahl C-G. Pharmacokinetics and systemic activity of fluticasone via Diskus and pMDI, and of budesonide via Turbuhaler. Br J Clin Pharmacol. 2001;52(5):52938. 


\section{Supplementary material}

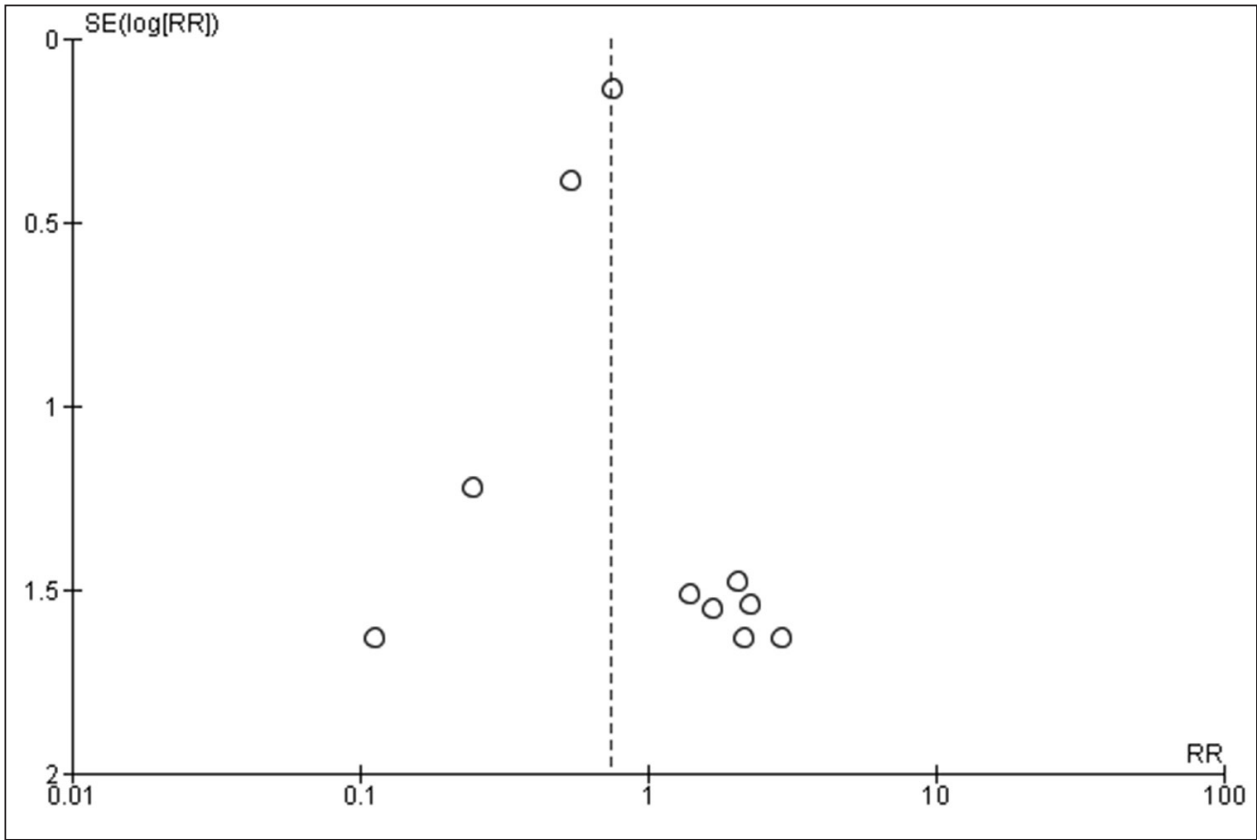

Figure S1 Funnel plot of RCTs suggesting no significant publication bias.

Search strategy (complete copied electronic search sequence)

((corticosteroid* OR beclomethasone OR triamcinolone* OR flunisolide OR budesonide OR fluticasone OR mometasone OR ciclesonide) AND (inhal* OR bronchodilat*) AND pneumoni* AND (los OR hospitali* OR ventilat* OR "length of stay")) NOT MEDLINE[sb] PubMed 15 August 2015 $=17$

\begin{tabular}{|c|c|c|c|}
\hline \multicolumn{4}{|c|}{ Ovid MEDLINE (R) In-Process \& Other Non-Indexed Citations and Ovid MEDLINE (R) 1946 to Present } \\
\hline & Searches & Results & Search Type \\
\hline 1 & $\begin{array}{l}\text { (beclomethasone or triamcinolone* or flunisolide or budesonide or fluticasone or } \\
\text { mometasone or ciclesonide).mp. }\end{array}$ & 21486 & Advanced \\
\hline 2 & exp glucocorticoids/ or 1 & 174737 & Advanced \\
\hline 3 & exp pneumonia/ or pneumoni*.mp. & 177636 & Advanced \\
\hline 4 & 2 and 3 & 3240 & Advanced \\
\hline 5 & (inhal* or ics).mp. or administration, inhalation/ & 139726 & Advanced \\
\hline 6 & 4 and 5 & 308 & Advanced \\
\hline 7 & $\begin{array}{l}\text { limit } 6 \text { to ("young adult (19 to } 24 \text { years)" or "adult ( } 19 \text { to } 44 \text { years)" or "young adult and } \\
\text { adult ( } 19-24 \text { and } 19-44) \text { " or "middle age ( } 45 \text { to } 64 \text { years)" or "middle aged ( } 45 \text { plus years)" or } \\
\text { "all aged ( } 65 \text { and over)" or "aged ( } 80 \text { and over)") }\end{array}$ & 134 & Advanced \\
\hline 8 & $\begin{array}{l}6 \text { and adult*.mp. [mp=title, abstract, original title, name of substance word, subject } \\
\text { heading word, keyword heading word, protocol supplementary concept word, rare } \\
\text { disease supplementary concept word, unique identifier] }\end{array}$ & 77 & Advanced \\
\hline 9 & ( & 145 & Advanced \\
\hline 10 & $\begin{array}{l}\text { limit } 9 \text { to (clinical trial, all or clinical trial, phase i or clinical trial, phase ii or clinical trial, } \\
\text { phase iii or clinical trial, phase iv or clinical trial or comparative study or controlled clinical } \\
\text { trial or evaluation studies or meta analysis or multicenter study or observational study or } \\
\text { randomized controlled trial) }\end{array}$ & 56 & Advanced \\
\hline 11 & $\begin{array}{l}9 \text { and ("case adj control*" or observational* or cohort* or retrospective* or prospective*). } \\
\text { mp. }\end{array}$ & 30 & Advanced \\
\hline 12 & 10 or 11 & 72 & Advanced \\
\hline 13 & limit 12 to $y r=" 1993-2015^{\prime \prime}$ & 65 & \\
\hline
\end{tabular}

Manually excluded studies on COPD patients; Central=48, same strategy 1993-2015; Embase 1988 to 2015. 


\begin{tabular}{|c|c|c|c|}
\hline \# & Searches & Results & Search Type \\
\hline 1 & $\begin{array}{l}\text { (beclomethasone or triamcinolone* or flunisolide or budesonide or fluticasone or } \\
\text { mometasone or ciclesonide).mp. }\end{array}$ & 46276 & Advanced \\
\hline 2 & exp pneumonia/ or pneumoni*.mp. & 262692 & Advanced \\
\hline 3 & $\begin{array}{l}\text { exp asthma/ or asthma*.mp. or copd.mp. or "chronic obstructive".mp. or pulmonary } \\
\text { disease, chronic obstructive/ }\end{array}$ & 264244 & Advanced \\
\hline 4 & exp glucocorticoid/ih & 9549 & Advanced \\
\hline 5 & $\begin{array}{l}\text { ( } 1 \text { and inhal*.mp.) or } 4 \text { [mp=title, abstract, heading word, drug trade name, original title, } \\
\text { device manufacturer, drug manufacturer, device trade name, keyword] }\end{array}$ & 16826 & Advanced \\
\hline 6 & 2 and 3 and 5 & 830 & Advanced \\
\hline 7 & limit 6 to (adult $<18$ to 64 years $>$ or aged $<65+$ years $>$ ) & 235 & Advanced \\
\hline 8 & $\begin{array}{l}\text { exp case control study/ or exp case study/ or exp clinical trial/ or exp "clinical trial (topic)"/ } \\
\text { or exp major clinical study/ or exp prospective study/ or exp retrospective study/ }\end{array}$ & 3160131 & Advanced \\
\hline 9 & 7 and 8 & 138 & Advanced \\
\hline 10 & $\begin{array}{l}7 \text { and (cohort* or observation*).mp. [mp=title, abstract, heading word, drug trade name, } \\
\text { original title, device manufacturer, drug manufacturer, device trade name, keyword] }\end{array}$ & 25 & Advanced \\
\hline 11 & 9 or 10 & 143 & Advanced \\
\hline 12 & remove duplicates from 11 & 140 & Advanced \\
\hline 13 & limit 12 to $y r=" 1993-2015^{\prime \prime}$ & 140 & \\
\hline
\end{tabular}

Manually excluded studies on COPD patients

Web of Science

$\mathrm{TS}=\left(\right.$ beclomethasone OR triamcinolone* $\mathrm{OR}$ flunisolide OR budesonide OR fluticasone OR mometasone OR ciclesonide OR (inhal ${ }^{*}$ OR ics) SAME (corticosteroid* OR steroid OR glucocorticoid*)) AND TS=(trial* OR random* OR cohort* OR prospective* OR retrospective* OR observation* OR "case contro*" OR study OR studies) AND TS=(asthma* OR copd OR"chronic obstructive" OR pneumoni*) NOT TI=(child* OR baby OR babies OR infant* OR newborn OR neonat* OR child* OR pediatr* OR paediatr* OR adolescen* OR teen*) 292

1993-2015

Scopus

TITLE-ABS-KEY ( ( beclomethasone OR triamcinolone* OR flunisolide OR budesonide OR fluticasone OR mometasone OR ciclesonide OR ( inhal* W/5 (corticosteroid* OR ics OR steroid* OR glucocorticoid*))) ) AND TITLE-ABS-KEY (( trial* OR random* OR cohort* OR prospective* OR retrospective* OR observation*) AND (asthma* OR copd OR "chronic obstructive") AND pneumoni*) AND NOT TITLE-ABS-KEY ( ( child* OR baby OR babies OR infant* OR newborn OR neonat* OR child* OR pediatr* OR paediatr* OR adolescen* OR teen*) ) AND PUBYEAR > 1992483

\begin{tabular}{|c|c|c|c|c|c|c|c|c|c|c|}
\hline \multirow[b]{2}{*}{ Study or Subgroup } & \multicolumn{2}{|l|}{ ICS } & \multicolumn{2}{|c|}{ Non ICS } & \multirow[b]{2}{*}{ Weight } & \multirow{2}{*}{$\begin{array}{c}\text { Risk Ratio } \\
\text { M-H, Random, } 95 \% \mathrm{Cl}\end{array}$} & \multirow{2}{*}{\multicolumn{4}{|c|}{$\begin{array}{c}\text { Risk Ratio } \\
\text { M-H, Random, 95\% Cl }\end{array}$}} \\
\hline & Events & Total & Events & Total & & & & & & \\
\hline ADA103575 & 2 & 544 & 0 & 181 & $11.8 \%$ & $1.67[0.08,34.62]$ & & & & \\
\hline Busse 2012 & 3 & 519 & 0 & 103 & $12.4 \%$ & $1.40[0.07,26.90]$ & & & & \\
\hline Busse 2014 & 1 & 232 & 2 & 115 & $19.0 \%$ & $0.25[0.02,2.70]$ & & & & \\
\hline Corren 2007 & 1 & 244 & 0 & 236 & $10.6 \%$ & $2.90[0.12,70.88]$ & & & & \\
\hline Karpel 2007 & 2 & 85 & 0 & 38 & $11.9 \%$ & $2.27[0.11,46.12]$ & & & & \\
\hline Maspero 2013 & 0 & 424 & 1 & 142 & $10.6 \%$ & $0.11[0.00,2.74]$ & & & & \\
\hline Noonan 2006 & 1 & 348 & 0 & 248 & $10.6 \%$ & $2.14[0.09,52.32]$ & & & & \\
\hline$O^{\prime}$ Byrne 2011 & 15 & 5437 & 12 & 2335 & $0.0 \%$ & $0.54[0.25,1.15]$ & & & & \\
\hline Sheffer 2005 & 86 & 3630 & 113 & 3591 & $0.0 \%$ & $0.75[0.57,0.99]$ & & & & \\
\hline Woodcock 2011 & 5 & 545 & 0 & 101 & $13.0 \%$ & $2.05[0.11,36.88]$ & & & & \\
\hline Total (95\% Cl) & & 2941 & & 1164 & $100.0 \%$ & $0.99[0.35,2.81]$ & & & & \\
\hline Total events & 15 & & 3 & & & & & & & \\
\hline \multicolumn{7}{|c|}{$\begin{array}{l}\text { Heterogeneity: } \mathrm{Tau}^{2}=0.00 ; \mathrm{Chi}^{2}=4.44, \mathrm{df}=7(\mathrm{P}=0.73) ; \mathrm{I}^{2}=0 \% \\
\text { Test for overall effect: } Z=0.02(\mathrm{P}=0.99)\end{array}$} & 0.01 & $\begin{array}{l}1 \\
0.1 \\
\text { Favours ICS }\end{array}$ & \begin{tabular}{|c|}
10 \\
Favours Non-ICS
\end{tabular} & 100 \\
\hline
\end{tabular}

Figure S2 Sensitivity analysis for RCTs: Without Sheffer 2005 and O’Byrne 2011 studies data. 


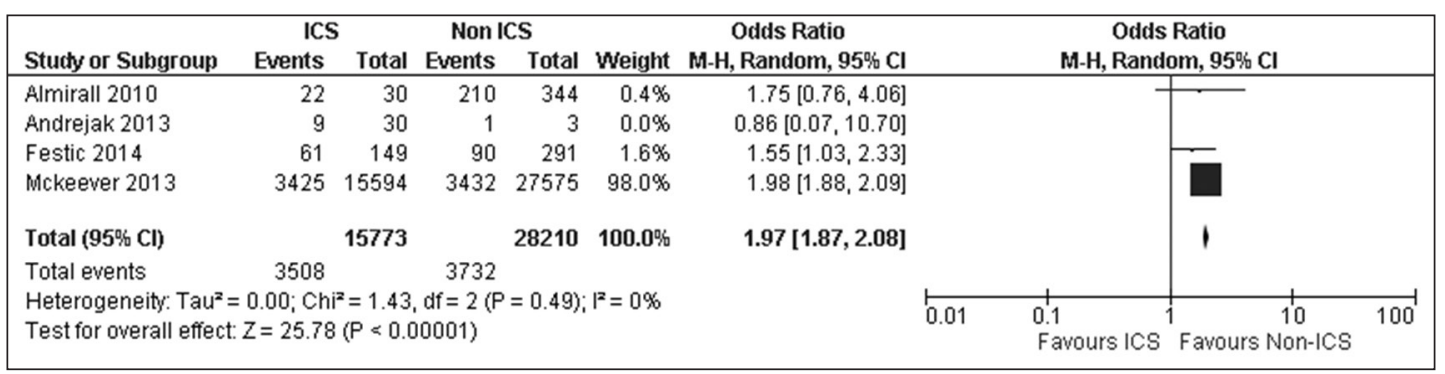

Figure S3 Sensitivity analyses for observational studies: Without Andrejak 2013 (NTM) study data.

\section{Quality assessment scale for cohort studies}

Note: A study can be awarded a maximum of one star for each numbered item within the Selection and Exposure categories. A maximum of two stars can be given for Comparability.

Selection

1) Representativeness of the exposed cohort

A. Truly representative of patients with asthma on ICS in the community 燐

B. Somewhat representative of patients with asthma on ICS in the community

C. Selected group of participants

D. No description of the derivation of the cohort

2) Selection of the non-exposed cohort

A. Drawn from the same community as the exposed cohort

B. Drawn from a different source

C. No description of the derivation of the non-exposed cohort

3) Ascertainment of exposure (ICS)
A. Prescription, medical records 粠
B. Self-report
C. No description

4) Demonstration that outcome of interest (pneumonia) was not present at start of study
A. Yes 米
B. No

Comparability

5) Comparability of cohorts on the basis of the design or analysis: (a maximum of 2 stars can be allotted)
A. Adjusted analysis (age $=$ *, other ad- justments also $=$ **, i.e. Demographics, comorbidities, medications etc.)
B. Unadjusted

Note: If the relative risk for the exposure of interest is adjusted for the confounders listed, then the groups will be considered to be comparable on each variable used in the adjustment.

\section{Outcome}

6) Assessment of outcome (pneumonia)

A. Radiographic plus clinical diagnosis ${ }^{-}$

B. Clinical diagnosis only *

C. No description

Assessment of outcome (pneumoniarelated mortality, deaths in those with pneumonia)
A. Reported 粠

B. Not clear

Assessment of outcome (overall mortality, all deaths)
A. Reported 粠
B. Not clear

7) Was follow-up long enough for outcomes to occur
A. Yes ( $\geq 30$ days or hospitalization for pneumonia) 米
B. No

8) Adequacy of follow up of cohorts:

A. Adequate follow up: $>90 \%$ of subjects accounted for

B. Acceptable follow up: $>50 \%$ of subjects accounted for and unlikely to introduce bias or described 档 
C. Follow up rate at the end of the study was $<50 \%$ and no description of those lost

D. No description

\section{Quality assessment scale for case- control studies}

Note: A study can be awarded a maximum of one star for each numbered item within the Selection and Exposure categories. A maximum of two stars can be given for Comparability.

\section{Selection}

1) Is the case definition adequate (pneumonia)?
A. Clinical diagnosis 粠
B. No reference

2) Representativeness of the cases

A. All eligible cases over a defined period of time/catchment area 将

B. Appropriate sample of cases (random sample)

C. Not stated

3) Selection of controls

A. Same population as above (same community) 将

B. Hospital controls

C. No description

4) Definition of Controls
A. No current (recent) ICS use 犊
B. Not stated

Comparability

5) Comparability of cases and controls on the basis of the design or analysis

A. Study controls for age $=$ 档

B. Study controls for other factors = 燐, i.e. severity, comorbidities, etc.)

\section{Exposure}

6) Ascertainment of exposure (ICS)

A. Secure record (prescription, medical chart etc.) $)^{-}$

B. No description or not as above
7) Same method of ascertainment for cases and controls

A. Yes 粠

B. No

8) Non-Response rate

A. Same no consent rate (refusal) for both/all groups 米

B. Different no consent (refusal) rate non respondents described

\section{References for excluded studies:}

1. Study No. D589IL00001 Clinicaltrials.gov Identifier NCT01232348. [Accessed 2014 June 17]. Available from: http://www.astrazenecaclinicaltrials.com/Submission/View?id=1617.

2. Beasley RW, Donohue JF, Mehta R, Nelson HS, Clay M, Moton A, et al. Effect of once-daily indacaterol maleate/mometasone furoate on exacerbation risk in adolescent and adult asthma: a double-blind randomised controlled trial. BMJ Open. 2015;5(2):e006131.

3. Study No. D5890L00008 Clinicaltrials.gov Identifier NCT00242411. [Accessed 2014 June 17]. Available from: http://www.astrazenecaclinicaltrials.com/Submission/View?id=1523.

4. Study No.D5890L00009 Clinicaltrials.gov Identifier NCT00290264. [Accessed 2014 June 17]. Available from: http://www.astrazenecaclinicaltrials.com/Submission/View?id=1526.

5. Hojo M, Iikura M, Hirano S, Sugiyama H, Kobayashi N, Kudo K. Increased risk of nontuberculous mycobacterial infection in asthmatic patients using long-term inhaled corticosteroid therapy. Respirology. 2012;17(1):185-90.

6. Lin J, Kang J, Lee SH, Wang C, Zhou X, Crawford J, et al. Fluticasone furoate/vilanterol 200/25 mcg in Asian asthma patients: a randomized trial. Respir Med. 2015;109(1):44-53.

7. Bodzenta-Lukaszyk A, Dymek A, McAulay K, Mansikka H. Fluticasone/formoterol combination therapy is as effective as fluticasone/salmeterol in the treatment of asthma, but has a more rapid onset of action: an open-label, randomized study. BMC Pulm Med. 2011;11:28.

8. Bodzenta-Lukaszyk A, Pulka G, Dymek A, Bumbacea D, McIver T, Schwab B, et al. Efficacy and safety of fluticasone and formoterol in a single pressurized metered dose inhaler. Respir Med. 2011;105(5):674-82.

9. Study No. SAM 106538. Clinicaltrials.gov Identifier NCT00363480. [Accessed 2014 June 17]. Avail- 
able from: http://www.gsk-clinicalstudyregister. com/study/SAM\%20106538?study_ids=SAM\%20 106538\#ps.

10. Peters SP, Kunselman SJ, Icitovic N, Moore WC, Pascual R, Ameredes BT, et al. Tiotropium bromide step-up therapy for adults with uncontrolled asthma. N Engl J Med. 2010;363(18):1715-26.

11. Teichert M, Schermer T, van den Nieuwenhof L, De Smet PA, Wensing M. Prevalence of inappropriate prescribing of inhaled corticosteroids for respiratory tract infections in the Netherlands: a retrospective cohort study. NPJ Prim Care Respir Med. 2014;24:14086.

12. Woodcock A, Lotvall J, Busse WW, Bateman ED, Stone S, Ellsworth A, et al. Efficacy and safety of fluticasone furoate $100 \mathrm{mug}$ and 200 mug once daily in the treatment of moderate-severe asthma in adults and adolescents: a 24-week randomised study. BMC Pulm Med. 2014;14:113.

13. Corren J, Mansfield LE, Pertseva T, Blahzko V, Kaiser K. Efficacy and safety of fluticasone/formoterol combination therapy in patients with moderateto-severe asthma. Respir Med. 2013;107(2):18095.

14. Bleecker ER, Lotvall J, O’Byrne PM, Woodcock A, Busse WW, Kerwin EM, et al. Fluticasone furoatevilanterol 100-25 mcg compared with fluticasone furoate $100 \mathrm{mcg}$ in asthma: a randomized trial. J Allergy Clin Immunol Pract. 2014;2(5):553-61.

15. Nathan RA, D'Urzo A, Blazhko V, Kaiser K. Safety and efficacy of fluticasone/formoterol combination therapy in adolescent and adult patients with mild-to-moderate asthma: a randomised controlled trial. BMC Pulm Med. 2012;12:67.

16. Pearlman DS, LaForce CF, Kaiser K. Fluticasone/Formoterol combination therapy compared with monotherapy in adolescent and adult patients with mild to moderate asthma. Clin Ther. 2013;35(7):950-66.

17. Price D, Popov TA, Bjermer L, Lu S, Petrovic R, Vandormael K, et al. Effect of montelukast for treatment of asthma in cigarette smokers. J Allergy Clin Immunol. 2013;131(3):763-71.
18. Lee CH, Jang EJ, Hyun MK, Lee NR, Kim K, Yim JJ. Risk of hospital admission or emergency room visit for pneumonia in patients using respiratory inhalers: a case-crossover study. Respirology. 2013;18(7):1116-27.

19. Almirall J, Bolibar I, Serra-Prat M, Roig J, Hospital I, Carandell E, et al. New evidence of risk factors for community-acquired pneumonia: a population-based study. Eur Respir J. 2008;31(6):127484.

20. Almirall J, Bolibar I, Serra-Prat M, Palomera E, Roig J, Hospital I, et al. Relationship between the use of inhaled steroids for chronic respiratory diseases and early outcomes in community-acquired pneumonia. PLoS One. 2013;8(9):e73271.

21. O’Byrne PM, Pedersen S, Lamm CJ, Tan WC, Busse WW. Severe exacerbations and decline in lung function in asthma. Am J Respir Crit Care Med. 2009;179(1):19-24.

22. Study No. D589IL00001 Clinicaltrials.gov Identifier NCT01232335. [Accessed 2014 June 17]. Available from: http://www.astrazenecaclinicaltrials.com/Submission/View?id=558.

23. Pauwels RA, Pedersen S, Busse WW, Tan WC, Chen YZ, Ohlsson SV, et al. Early intervention with budesonide in mild persistent asthma: a randomised, double-blind trial. Lancet. 2003;361(9363):1071-6.

24. Almirall J, Bolibar I, Balanzo X, Gonzalez CA. Risk factors for community-acquired pneumonia in adults: a population-based case-control study. Eur Respir J. 1999;13(2):349-55.

25. Eurich DT, Lee C, Marrie TJ, Majumdar SR. Inhaled corticosteroids and risk of recurrent pneumonia: a population-based, nested case-control study. Clin Infect Dis. 2013;57(8):1138-44.

26. Farr BM, Bartlett CL, Wadsworth J, Miller DL. Risk factors for community-acquired pneumonia diagnosed upon hospital admission. British Thoracic Society Pneumonia Study Group. Respir Med. 2000;94(10):954-63. 